Stability of solutions of hydrodynamic equations describing the scaling limit of a massive piston in an ideal gas

This article has been downloaded from IOPscience. Please scroll down to see the full text article.

2004 Nonlinearity 17897

(http://iopscience.iop.org/0951-7715/17/3/009)

View the table of contents for this issue, or go to the journal homepage for more

Download details:

IP Address: 151.100.50.155

The article was downloaded on 09/05/2012 at 13:45

Please note that terms and conditions apply. 


\title{
Stability of solutions of hydrodynamic equations describing the scaling limit of a massive piston in an ideal gas
}

\author{
E Caglioti ${ }^{1}$, N Chernov ${ }^{2}$ and J L Lebowitz ${ }^{3}$ \\ 1 Dipartimento di Matematica, Università di Roma 'La Sapienza' Piazzale Aldo Moro 2, \\ 00185 Roma, Italy \\ 2 Department of Mathematics, University of Alabama at Birmingham, Alabama 35294, USA \\ ${ }^{3}$ Department of Mathematics, Rutgers University, NJ 08854, USA
}

Received 17 February 2003, in final form 2 February 2004

Published 27 February 2004

Online at stacks.iop.org/Non/17/897 (DOI: 10.1088/0951-7715/17/3/009)

Recommended by K Ohkitani

\begin{abstract}
We analyse the stability of stationary solutions of a singular Vlasov type hydrodynamic equation (HE). This equation was derived (under suitable assumptions) as the hydrodynamical scaling limit of the Hamiltonian evolution of a system consisting of a massive piston immersed in an ideal gas of point particles in a box. We find explicit criteria for global stability as well as a class of solutions that are linearly unstable for a dense set of parameter values. We present some numerical evidence that when the mechanical system (with a large number of particles) has initial conditions 'close' to stationary stable solutions of the HE, then it stays close to these solutions for a long time. On the other hand, if the initial state of the particle system is close to an unstable stationary solution of the HE, then the mechanical system diverges rapidly from that solution and later appears to develop long lasting periodic oscillations. We find similar (approximately periodic) solutions of the HE that are linearly stable.
\end{abstract}

PACS numbers: 05.20.Jj, 47.20.Ky

\section{Introduction}

The time evolution of a system consisting of a piston of mass $M$ moving parallel to the $x$-axis in a cube containing non-interacting point particles of unit mass has been studied extensively [CLS1,CLS2,CL, G, GF, GP, H, KBM,LPS,Li, PG]. After some rescaling of space and time (by the length of the cube), the problem reduces to that of a one-dimensional system with $N_{\mathrm{L}}\left(N_{\mathrm{R}}\right)$ particles in the interval $[0, X]$ (respectively, $[X, 1]$ ) where $X(t)$ is the position of the piston. The left (right) particles move freely between collisions with the wall at $x=0$ 
$(x=1)$ and the piston at $x=X(t)$. At the walls the velocities of the particles get reversed, while at $x=X(t)$ the outgoing velocity, $v^{\prime}$, is related to the incoming velocity, $v$, by the rules of elastic collisions [CLS2, CL, LPS],

$$
v^{\prime}=-\frac{M-1}{M+1} v+\frac{2 M}{M+1} V
$$

where $V$ is the incoming velocity of the piston. It follows from (1.1) that $N_{\mathrm{L}}$ and $N_{\mathrm{R}}$ as well as the total kinetic energy, $\frac{1}{2} \sum_{i=1}^{N} v_{i}^{2}+\frac{1}{2} M V^{2}\left(N=N_{\mathrm{L}}+N_{\mathrm{R}}\right)$, are conserved quantities. The dynamics of the system can be reduced to a billiard in a $(2 N+1)$-dimensional domain (polyhedron) (cf [CL]). It was shown in [LPS, CLS1, CLS2], under certain quite restrictive conditions on the initial distribution of gas particles, that, in the limit $N \rightarrow \infty, M \sim N^{2 / 3}$, the dynamics of the piston and the gas satisfy a closed system of Euler type hydrodynamic equations (HEs) for a time interval $(0, \tau)$ in which any particle had at most two collisions with the piston.

The origin of the scaling $M \sim N^{2 / 3}$ is as follows. For $N$ particles with velocities of $\mathrm{O}(1)$ distributed with a density of $\mathrm{O}(1)$ in a parallelepiped of length $L$ and cross-sectional area $A$, the number of particles colliding with the piston per unit (unrescaled) time, and hence the pressure (from each side), is proportional to $A$. To ensure that, on this timescale, the acceleration of the piston stays of $\mathrm{O}(1)$ as $L, A$ and $N \sim \mathrm{O}(A L)$ grow to infinity, it is necessary to make the mass of the piston grow as $A$. For a cube, this corresponds to $M \sim N^{2 / 3}$. In the rescaled units, the number of collisions experienced by the piston per unit time is $\mathrm{O}(N)$ independent of $A$, and the HEs hold for general $M$ as long as $M \sim N^{\alpha}, \alpha \in(0,1)$, i.e. when the kinetic energy of the piston becomes negligible compared with that of the gas. The time interval (in the scaled units) during which the derivation of the HE remains valid depends on $\alpha$ (getting larger as $\alpha \rightarrow 1$ ), see remarks 3 and 4 in section 4 of [CLS2]. For $\alpha=\frac{2}{3}$, this time interval is such that the piston suffers no more than two collisions with any gas particle. It is however not clear from the derivation to what extent those equations may actually approximate the real evolution of the particle system with large $N$, for longer times.

This led us to carry out extensive computer simulations of particle systems, with $M \sim N^{2 / 3}$ (precisely, $M=2 N^{2 / 3}$ ), $N$ as large as $27 \times 10^{6}[\mathrm{CL}]$, and initial conditions for which the HEs have a trivial stationary solution $X(t)=0.5$ and $V(t)=0$ for all $t>0$. We found nevertheless that for certain initial velocity distributions (see later) the trajectory of the piston diverged greatly from these values after a few collision times. In particular, it was observed in these simulations that the motion of the piston, after experiencing large seemingly random fluctuations, quickly converges to a more stable regime, in which the piston and the gas undergo regular (slowly damped) oscillations lasting many collision times. The parameters of these oscillations (the period, the amplitude, and the rate of damping) seem to depend very little on the number of point particles and some other details of the initial distributions.

It is reasonable to conjecture that the behaviour of a mechanical system for finite but large $N$ is related to the solution of the HE subjected to random fluctuations arising from the discrete nature of the gas particles. The large deviations of the motion of the mechanical system from the solution of HE, observed in [CL], may thus be due to the instability of the HE for the initial conditions considered; $\mathrm{cf}$ the discussion of hydrodynamic fluctuation theory [S].

This interpretation requires that the HE describe the limit (as $N \rightarrow \infty$ ) of the mechanical evolution of the system for times much longer than those for which they were derived in [CLS2]. This in turn would imply that when the solution of the HEs is stable, the mechanical evolution of the system will remain close to that solution for arbitrarily long times as $N \rightarrow \infty$. We note further that by choosing $\alpha$ in $M \sim N^{\alpha}$ close to 1 , the time for which the rigorous derivation of the HE remains valid can be made arbitrary large. Motivated by these considerations, we investigate here stability properties of a special class of stationary solution of the HE. These 
are of independent intrinsic interest since they represent, as far as we know, a first example of a rigorous derivation of non-trivial continuum equations from a Hamiltonian system (without any stochasticity).

This paper is organized as follows. In section 2, we state the HEs and discuss their structure. In section 3, we prove (rigorously) global stability for a class of stationary solution. We note that simulations of the mechanical systems with such distributions in [CL] indeed yield a piston trajectory that remains close to the solution of the HE, with $V(t) \sim 0$ for all $t>0$. In section 4 , we use a perturbative analysis to find sufficient conditions for linear instability (our arguments here are heuristic, but the conclusions are in good agreement with simulations). In section 5, we investigate a particular family of stationary solutions, which includes those used in the [CL] simulations, and show that, according to the criteria developed in section 4, it contains both linearly stable and unstable ones, alternating in a very intricate manner. We also describe there numerical solutions of the HE for two initial conditions obtained by adding the same perturbation to a linearly stable state and a linearly unstable stationary state. After a short initial time in which the solutions behave similarly, they exhibit interesting differences (figures 3-5). In section 6, we compare a mechanical trajectory of the piston with a solution of the HE with initial conditions obtained from those used for the simulation of the mechanical system by a small perturbation. The two trajectories look startlingly similar, thus confirming our previous analysis. After an initial period of 10-15 recollision times, both trajectories evolve almost periodically for another 15-20 recollision times, which is much longer than the time for which the equations were derived in [CLS2] but sufficiently short so that the periodic motion of the mechanical system has no visible damping. These observations suggest the existence of a periodic solution of the HE, and we then carry out an approximate analytic construction of such periodic solutions.

\section{Hydrodynamic equations}

Let $X(t) \in(0,1)$ be the position of the piston at time $t$ and $V(t)$ its velocity. We denote the continuum density of the gas in $[0,1] \times \mathbb{R}$ by a function $p(x, v, t)$. The HEs describing the time evolution of this continuum fluid plus piston system are as follows.

(H1) Free motion. Inside the container the density satisfies the standard continuity equation for a non-interacting particle system without external forces:

$$
\left(\frac{\partial}{\partial t}+v \frac{\partial}{\partial x}\right) p(x, v, t)=0
$$

for all $x \in(0,1)$ except $x=0, x=1$, and $x=X(t)$.

Equation (2.1) has a simple solution,

$$
p(x, v, t)=p(x-v s, v, t-s)
$$

for $0<s<t$ such that $x-v r \notin\{0, X(t-r), 1\}$ for all $r \in(0, s)$. Equation (2.2) has one advantage over (2.1): it applies to all points $(x, v)$, including those where the function $p$ is not differentiable or even continuous.

(H2) Collisions with the walls. At the walls, $x=0$ and $x=1$, we have

$$
\begin{aligned}
& p(0, v, t)=p(0,-v, t), \\
& p(1, v, t)=p(1,-v, t) .
\end{aligned}
$$


(H3) Collisions with the piston. At the piston, $x=X(t)$, we have (this is obtained from (1.1) when $M \rightarrow \infty$ )

$\begin{array}{lr}p(X(t)-0, v, t)=p(X(t)-0,2 V(t)-v, t) & \text { for } v<V(t), \\ p(X(t)+0, v, t)=p(X(t)+0,2 V(t)-v, t) & \text { for } v>V(t),\end{array}$

where $v$ represents the velocity after the collision and $2 V(t)-v$ that before the collision; and

$$
X(t)=X(0)+\int_{0}^{t} V(s) \mathrm{d} s
$$

is the (deterministic) position of the piston.

It remains for us to describe the evolution of $V(t)$ (which we take to be left continuous). Suppose the piston's position at time $t$ is $X$ and its velocity $V$. The piston is affected by the fluid at $(x, v)$ exerting pressure on it from the right $(x=X+0$ and $v<V)$ and from the left ( $x=X-0$ and $v>V$ ). Accordingly, we define the density of the fluid in contact with the piston before a collision ('density on the piston') by

$$
P(v, t ; X, V)= \begin{cases}p(X+0, v, t) & \text { if } v<V \\ p(X-0, v, t) & \text { if } v>V .\end{cases}
$$

(H4) Piston's velocity. The velocity $V=V(t)$ of the piston satisfies the equation

$$
\int_{-\infty}^{\infty}(v-V)^{2} \operatorname{sgn}(v-V) P(v, t ; X, V) \mathrm{d} v=0 .
$$

The origin of equations (H1)-(H3) in the particle system is clear. (H4) is essentially a force balance equation - because the rate of collision of the piston with particles on either side and the consequent force on the piston much larger than the mass of the piston when $N / M \rightarrow \infty$, $V$ adjusts instantaneously to make the forces from the two sides balance exactly. The system of (hydrodynamic) equations (H1)-(H4) is now closed and, given initial conditions, satisfying (2.3)-(2.8) at $t=0$, completely determines the functions $X(t), V(t)$, and $p(x, v, t)$ for $t>0$. When the initial conditions do not satisfy these equations, one has to imagine that they become satisfied instantaneously for $t=0+$. The existence and uniqueness of solutions of (H1)-(H4) were proven, under general conditions, in [CLS1, CLS2]. We need only to assume that $p(x, v, 0)$ is bounded, piecewise differentiable, and either has a compact support in the $x, v$ plane or decays fast enough as $|v| \rightarrow \infty$. We also require that $\int p(x, v, 0) \mathrm{d} v>0$ for all $x$.

The HEs, like the Vlasov equations for plasmas, are time-reversible (see [P, MP]). They preserve the classical integrals of motion. The mass of the fluid to the left and to the right of the piston as well as the total kinetic energy of the fluid remain constant along any solution.

(D1) Mass conservation

$$
\mathcal{M}_{\mathrm{L}}=\int_{0}^{X(t)} \int p(x, v, t) \mathrm{d} v \mathrm{~d} x, \quad \mathcal{M}_{\mathrm{R}}=\int_{X(t)}^{1} \int p(x, v, t) \mathrm{d} v \mathrm{~d} x .
$$

(D2) Energy conservation

$$
2 E=\iint v^{2} p(x, v, t) \mathrm{d} v \mathrm{~d} x .
$$


Just like the energy, the total momentum of the fluid, $\iint v p(x, v, t) \mathrm{d} v \mathrm{~d} x$, is also left unchanged by the fluid-piston interaction, but unlike the energy, the momentum changes due to reflections at the walls. The reason why the piston itself does not contribute to the total momentum and energy of the system in this model is due to the fact that its mass and energy vanish when divided by $N$, in the limit $N \rightarrow \infty$. (The mass, energy, and momentum of the fluid all correspond to the original quantities in the particle system divided by $N$.)

The HEs define a dynamics on the domain $G:=\{(x, v): 0 \leqslant x \leqslant 1\}$ in which every point $(x, v) \in G$ moves freely with constant velocity and collides elastically with the walls and the piston. Denote by $\left(x_{t}, v_{t}\right)$ the position and velocity of an arbitrary point at time $t \geqslant 0$. Then (H1) translates into $\dot{x}_{t}=v_{t}$ and $\dot{v}_{t}=0$ whenever $x_{t} \notin\{0,1, X(t)\},(\mathrm{H} 2)$ becomes $\left(x_{t+0}, v_{t+0}\right)=\left(x_{t-0},-v_{t-0}\right)$ whenever $x_{t-0} \in\{0,1\}$, and $(\mathrm{H} 3)$ gives

$$
\left(x_{t+0}, v_{t+0}\right)=\left(x_{t-0}, 2 V(t)-v_{t-0}\right)
$$

whenever $x_{t-0}=X(t)$. Note that the point $\left(x_{t}, v_{t}\right)$ moves in $G$ and reflects at the walls and the piston as if these had infinite mass.

The motion of points in $G$ is described by a one-parameter family of transformations $F^{t}: G \rightarrow G$ defined by $F^{t}\left(x_{0}, v_{0}\right)=\left(x_{t}, v_{t}\right)$ for $t>0$. We will also write $F^{-t}\left(x_{t}, v_{t}\right)=$ $\left(x_{0}, v_{0}\right)$. According to $(\mathrm{H} 1)-(\mathrm{H} 3)$, the density, $p(x, v, t)$, satisfies a simple equation,

$$
p\left(x_{t}, v_{t}, t\right)=p\left(F^{-t}\left(x_{t}, v_{t}\right), 0\right)=p\left(x_{0}, v_{0}, 0\right)
$$

for all $t \geqslant 0$. It is easy to see that for each $t>0$ the map $F^{t}$ is one-to-one and preserves area, i.e. $\operatorname{det}\left|D F^{t}(x, v)\right|=1$. Hence, the family $F^{t}$ describes an incompressible flow on $G$ and consequently:

(D3) Incompressibility. For any $a<b$ the Lebesgue measures (areas) of the sets,

$$
\{(x, v): a<p(x, v, t)<b, 0<x<X(t)\}
$$

and

$$
\{(x, v): a<p(x, v, t)<b, X(t)<x<1\}
$$

remain constant in time.

A particular case in which it is possible to solve equations (H1)-(H4) analytically is when the initial distribution is stationary. This happens when $p(x, v, 0)$ satisfies two conditions.

(S1) Uniformity and symmetry. The initial density $p(x, v, 0)=p(x, v)$ is of the form

$$
p(x, v)= \begin{cases}p_{\mathrm{L}}(|v|) & \text { for } x<X_{0} \\ p_{\mathrm{R}}(|v|) & \text { for } x>X_{0}\end{cases}
$$

for all $v$ and $X(0)=X_{0}$.

(S2) Pressure balance. The pressure on the piston from both sides is equal:

$$
P_{\mathrm{L}}:=2 \int_{0}^{\infty} v^{2} p_{\mathrm{L}}(v) \mathrm{d} v=P_{\mathrm{R}}:=2 \int_{0}^{\infty} v^{2} p_{\mathrm{R}}(v) \mathrm{d} v .
$$

Under conditions (S1)-(S2) the equations (H1)-(H4) have a simple solution-the system remains frozen in its initial state:

$$
X(t) \equiv X_{0}, \quad V(t) \equiv 0, \quad p(x, v, t) \equiv p(x, v, 0)
$$

for all $t>0$

We will analyse in the next three sections the stability of this stationary solution. Note that there is no requirement on the form of $p_{\mathrm{L}}(|v|)$ or $p_{\mathrm{R}}(|v|)$; all that is required is a balance of forces (2.10). 


\section{Globally stable solutions}

Here, we prove that stationary solutions $p(x, v)$ satisfying $(\mathrm{S} 1)-(\mathrm{S} 2)$ and an additional monotonicity requirement,

$$
p_{\mathrm{L}}\left(\left|v_{1}\right|\right) \geqslant p_{\mathrm{L}}\left(\left|v_{2}\right|\right) \quad \text { and } \quad p_{\mathrm{R}}\left(\left|v_{1}\right|\right) \geqslant p_{\mathrm{R}}\left(\left|v_{2}\right|\right)
$$

for all $\left|v_{1}\right| \leqslant\left|v_{2}\right|$ are globally stable. This criterion is very similar to the stability criteria for the Vlasov equation described by Penrose [P] and by Marchioro and Pulvirenti [MP].

Before we state our result, we introduce some notation. Denote by $\|\cdot\|$ the following special norm on the space of functions on $G$ :

$$
\|f(x, v)-g(x, v)\|=\iint|f(x, v)-g(x, v)|\left(1+v^{2}\right) \mathrm{d} v \mathrm{~d} x .
$$

Theorem 3.1. Let $p(x, v)$ satisfy $(S 1),(S 2)$, and (3.1). Then for any $\varepsilon>0$, there exists $a$ $\delta>0$ such that if the initial density, $p(x, v, 0)$, satisfies $\|p(x, v, 0)-p(x, v)\|<\delta$, and $X(0)=X_{0}$, then

(i) $\|p(x, v, t)-p(x, v)\|<\varepsilon$;

(ii) $|X(t)-X(0)|<\varepsilon$

for all $t>0$.

Proof. Our theorem is an almost straightforward consequence of the stability theorem ${ }^{4}$ by Marchioro and Pulvirenti [MP] (in particular, when $p_{\mathrm{L}}=p_{\mathrm{R}}$, then (i) is exactly their theorem), and so we only outline the argument here.

It is clear from (2.9) that, given the position of the piston, $X$, and values of the areas of the level sets defined in (D3), the minimal possible value of the total energy for any phase-space density, $\pi(x, v)$, is attained when $\pi(x, v)$ is uniform in $x$ and monotonically decreasing in $|v|$ in each compartment.

Consider first the case where $\pi(x, v)$ has, in each compartment, the same area of the level sets as some $p(x, v)$ satisfying $(\mathrm{S} 1)$ and $(\mathrm{S} 2)$. Then the minimum of the energy when the piston position is $X$ is attained when

and

$$
\pi(x, v)=p_{\mathrm{L}}\left(\frac{v X}{X_{0}}\right), \quad 0<x<X
$$

$$
\pi(x, v)=p_{\mathrm{R}}\left(\frac{v(1-X)}{1-X_{0}}\right), \quad X<x<1 .
$$

The minimal total energy is then

$$
\begin{aligned}
2 E_{\min }(t) & =\int_{0}^{\infty} \int_{0}^{X} v^{2} \pi(x, v) \mathrm{d} x \mathrm{~d} v+\int_{0}^{\infty} \int_{X}^{1} v^{2} \pi(x, v) \mathrm{d} x \mathrm{~d} v \\
& =\frac{X_{0}^{3}}{X^{2}} \int_{0}^{\infty} v^{2} p_{\mathrm{L}}(v) \mathrm{d} v+\frac{\left(1-X_{0}\right)^{3}}{(1-X)^{2}} \int_{0}^{\infty} v^{2} p_{\mathrm{R}}(v) \mathrm{d} v
\end{aligned}
$$

(we used a change of variable $u=v X / X_{0}$ in the first integral and $u=v(1-X) /\left(1-X_{0}\right)$ in the second one). Using the pressure balance (2.10) and denoting $P=P_{\mathrm{L}}=P_{\mathrm{R}}$ gives

$$
E_{\min }(t)=\frac{P}{2}\left(\frac{X_{0}^{3}}{X^{2}}+\frac{\left(1-X_{0}\right)^{3}}{(1-X)^{2}}\right) .
$$

\footnotetext{
4 We note that the stability theorem in [MP] is stated in the $L_{1}$ norm but is, in fact, proven in the (3.2) norm. It therefore needs additional conditions on the space of densities that make these two norms equivalent.
} 
Consider now the above expression as a function of $X$. Its minimum is attained at the point where $\mathrm{d} E_{\min } / \mathrm{d} X=0$, i.e.

$$
\frac{X_{0}^{3}}{X^{3}}=\frac{\left(1-X_{0}\right)^{3}}{(1-X)^{3}}
$$

which is only possible if $X=X_{0}$. Therefore, the state $X=X_{0}$ provides a unique minimum of the total energy function under the incompressibility constraint (D3). Any deviation of $X$ from $X_{0}$ would result in an increase in the total energy. Since only a small increase of the total energy is allowed by a $\delta$-perturbation of the initial density, then only small deviations of $X$ from $X_{0}$ are permitted. This proves claim (ii) and also implies that $E_{\mathrm{L}}$ and $E_{\mathrm{R}}$ remain at all times very close to the values corresponding to the unperturbed density. We can then apply the stability theorem of Marchioro and Pulvirenti [MP] and obtain (i).

This proves the theorem in the case where $p(x, v, 0)$ has exactly the same area of the level sets, in each compartment, as $p(x, v)$. For other perturbed initial densities, $p(x, v, 0)$, the above estimates only hold approximately, and our results then follow by standard approximation techniques.

Suppose, for example, that the initial density, $p(x, v, 0)$, satisfies (S1) and (3.1), but (S2) only holds approximately: $\left|P_{\mathrm{L}}-P_{\mathrm{R}}\right|=\Delta$ with a small $\Delta>0$. This is a particular case of our theorem, and hence the piston will remain $\varepsilon$-close to its initial position, $X_{0}$, at all times. Interestingly, we can estimate, in terms of $\Delta$, how far the piston can swing from its initial position. Indeed, the piston can move as long as

$\frac{X_{0}^{3}}{X(t)^{2}} \int_{0}^{\infty} v^{2} p(X(t)-0, v, t) \mathrm{d} v+\frac{\left(1-X_{0}\right)^{3}}{(1-X(t))^{2}} \int_{0}^{\infty} v^{2} p(X(t)+0, v, t) \mathrm{d} v \leqslant 2 E(0)$

where $X_{0}=X(0)$, as before, and $E(0)$ is the initial total energy:

$$
2 E(0)=X_{0} \int_{0}^{\infty} v^{2} p_{\mathrm{L}}(v) \mathrm{d} v+\left(1-X_{0}\right) \int_{0}^{\infty} v^{2} p_{\mathrm{R}}(v) \mathrm{d} v .
$$

By simple calculations one obtains, to the leading order of $\Delta$, the following bound on the piston displacements:

$$
\left|X(t)-X_{0}\right| \leqslant \frac{2 \Delta}{3 P_{\mathrm{L}}}\left(\frac{1}{X_{0}}+\frac{1}{1-X_{0}}\right)^{-1}+\mathrm{O}\left(\Delta^{2}\right) .
$$

\section{Perturbative analysis}

Here, we analyse the linear stability of the solutions of the HE corresponding to initial densities $p(x, v, 0)$ satisfying the following stricter version of (S1)-(S2).

(S3) Full uniformity and symmetry. The initial density, $p(x, v, 0)$, is uniform in $x$ across the entire cylinder, i.e. $p(x, v, 0)=p_{0}(|v|)$ for all $v$ and $0<x<1$.

We also assume that the piston is initially at the midpoint, $X(0)=0.5$. Of course, under the conditions (S3), the HEs (H1)-(H4) have a simple stationary solution (2.11). On the other hand, we no longer assume monotonicity (3.1).

We use perturbative analysis to investigate the stability of the stationary solution (2.11). Our arguments are essentially heuristic: we perturb the initial density (by $\varepsilon$ ) in the $L^{1}$ norm (or, equivalently, in the (3.2) norm) and then expand all the equations in $\varepsilon$ up to first order and ignore terms of higher order. 
From now on we denote by $p_{0}(v)=p_{0}(|v|)$ an initial density satisfying (S3) and by $p(x, v, 0)$ a perturbed initial density, which we write as

$$
p(x, v, 0)=p_{0}(|v|)+\varepsilon p_{1}(x, v, 0)
$$

where $\varepsilon$ is small and $\left\|p_{1}(x, v, 0)\right\|=1$. For $t>0$, we decompose the density, $p(x, v, t)$, as

$$
p(x, v, t)=p_{0}(|v|)+\varepsilon p_{1}(x, v, t) .
$$

We also set $p_{1}(x, v, t)=p_{\mathrm{L}}(x, v, t)$ for $x<X(t)$ and $p_{1}(x, v, t)=p_{\mathrm{R}}(x, v, t)$ for $x>X(t)$.

According to (2.8), the velocity, $V(t)$, of the piston is given by

$\int_{V}^{\infty}(v-V)^{2}\left[p_{0}(v)+\varepsilon p_{\mathrm{L}}(X, v, t)\right] \mathrm{d} v=\int_{-\infty}^{V}(v-V)^{2}\left[p_{0}(v)+\varepsilon p_{\mathrm{R}}(X, v, t)\right] \mathrm{d} v$

where $X=X(t)$ is the position of the piston. Expanding in $\varepsilon$ and ignoring terms of order o $(\varepsilon)$ gives

$$
V(t)=\varepsilon \frac{\int_{0}^{\infty} v^{2} p_{\mathrm{L}}(X, v, t) \mathrm{d} v-\int_{-\infty}^{0} v^{2} p_{\mathrm{R}}(X, v, t) \mathrm{d} v}{4 \int_{0}^{\infty} v p_{0}(v) \mathrm{d} v} .
$$

Integrating by parts, we obtain (for piecewise smooth $p_{0}$ )

$$
2 \int_{0}^{\infty} v p_{0}(v) \mathrm{d} v=-\int_{0}^{\infty} v^{2} p_{0}^{\prime}(v) \mathrm{d} v .
$$

We define

$$
h(v)=-p_{0}^{\prime}(v) \quad \text { for } v>0
$$

and for the sake of completeness set $h(-v)=h(v)$. Then

$$
V(t)=\varepsilon \frac{\int_{0}^{\infty} v^{2} p_{\mathrm{L}}(X, v, t) \mathrm{d} v-\int_{-\infty}^{0} v^{2} p_{\mathrm{R}}(X, v, t) \mathrm{d} v}{2 \int_{0}^{\infty} v^{2} h(v) \mathrm{d} v} .
$$

When $p_{0}(v)$ is not differentiable, we interpret $-h(v)$ as the generalized derivative of $p_{0}(v)$ Denote by $\langle\cdot\rangle_{+}$the integration $\int_{0}^{\infty} \cdot \mathrm{d} v$ and by $\langle\cdot\rangle_{-}$the integration $\int_{-\infty}^{0} \cdot \mathrm{d} v$. Then

$$
V(t)=\varepsilon \frac{\left\langle v^{2} p_{\mathrm{L}}(X, v, t)\right\rangle_{+}-\left\langle v^{2} p_{\mathrm{R}}(X, v, t)\right\rangle_{-}}{2\left\langle v^{2} h(v)\right\rangle_{+}} .
$$

The density of the gas after interaction with the piston is given by the formulae (2.5), which imply

$$
\begin{aligned}
p(X-0,-v, t) & =p(X-0, v+2 V, t) \\
& =p_{0}(v+2 V)+\varepsilon p_{\mathrm{L}}(X, v, t) \\
& =p_{0}(v)+2 V p_{0}^{\prime}(v)+\varepsilon p_{\mathrm{L}}(X, v, t) \\
& =p_{0}(v)-2 V h(v)+\varepsilon p_{\mathrm{L}}(X, v, t) .
\end{aligned}
$$

Here, we assume $v>0$ and ignore terms of order $\mathrm{o}(\varepsilon)$. Hence, the 'reflection rule' can be written as

$$
p_{\mathrm{L}}(X,-v, t)=p_{\mathrm{L}}(X, v, t)-h(v) \frac{\left\langle v^{2} p_{\mathrm{L}}(X, v, t)\right\rangle_{+}-\left\langle v^{2} p_{\mathrm{R}}(X, v, t)\right\rangle_{-}}{\left\langle v^{2} h(v)\right\rangle_{+}} .
$$

This expression suggests the introduction of new functions

$$
q_{\mathrm{R}, \mathrm{L}}(x, v, t)=\frac{p_{\mathrm{L}, \mathrm{R}}(x, v, t)}{h(v)}
$$


and

$$
\rho(v)=\frac{v^{2} h(v)}{\left\langle v^{2} h(v)\right\rangle_{+}} .
$$

The above expression for $p_{\mathrm{L}}(X,-v, t)$ can now be written as

$$
q_{\mathrm{L}}(X,-v, t)=q_{\mathrm{L}}(X, v, t)-\left\langle q_{\mathrm{L}}(X, v, t) \rho(v)\right\rangle_{+}+\left\langle q_{\mathrm{R}}(X, v, t) \rho(v)\right\rangle_{-} .
$$

Similarly, on the other side of the piston,

$$
q_{\mathrm{R}}(X, v, t)=q_{\mathrm{R}}(X,-v, t)+\left\langle q_{\mathrm{L}}(X, v, t) \rho(v)\right\rangle_{+}-\left\langle q_{\mathrm{R}}(X, v, t) \rho(v)\right\rangle_{-} .
$$

One can interpret these 'reflection rules' as follows: the functions $q_{\mathrm{L}}$ and $q_{\mathrm{R}}$ 'exchange' their average values with respect to the 'density', $\rho(v)$.

Note that $\rho(v)$ is normalized, so that $\langle\rho(v)\rangle_{+}=1$, but it is not necessarily positive (or even non-negative). On the other hand, when $\rho(v) \geqslant 0$, i.e. the unperturbed density, $p_{0}(|v|)$, is non-increasing, thus satisfying (3.1). In this case the stationary solution (2.11) is stable, as we already know by theorem 3.1. Here we recover this result by our perturbative analysis.

Theorem 4.1. The quantity

$$
Q=\iint \frac{q^{2}(x, v, t) \rho(v)}{|v|} \mathrm{d} x \mathrm{~d} v
$$

is constant in time, i.e. $\mathrm{d} Q / \mathrm{d} t=0$. Here, $q=q_{\mathrm{L}}$ for $x<X$ and $q=q_{\mathrm{R}}$ for $x>X$.

Proof. Clearly, $Q$ cannot change just due to the free motion of the gas or due to collisions with the walls, and so we only need to worry about collisions with the piston. The gas particles colliding with the piston during an infinitesimal interval $(t, t+\mathrm{d} t)$ lie in two triangles on the $x v$ plane: $X-v \mathrm{~d} t<x<X$ for $v>0$ and $X<x<X-v \mathrm{~d} t$ for $v<0$. The outgoing particles lie in similar symmetric triangles. Hence, during the interval $(t, t+\mathrm{d} t)$, the quantity $Q$ decreases by (up to the factor of $\mathrm{d} t$ )

$$
\begin{array}{r}
\int_{0}^{\infty}|v| \frac{q_{\mathrm{L}}^{2}(X, v, t) \rho(v)}{|v|} \mathrm{d} v+\int_{-\infty}^{0}|v| \frac{q_{\mathrm{R}}^{2}(X, v, t) \rho(v)}{|v|} \mathrm{d} v \\
=\left\langle q_{\mathrm{L}}^{2}(X, v, t) \rho(v)\right\rangle_{+}+\left\langle q_{\mathrm{R}}^{2}(X, v, t) \rho(v)\right\rangle_{-}
\end{array}
$$

and it increases by

$$
\begin{array}{r}
\int_{-\infty}^{0}|v| \frac{q_{\mathrm{L}}^{2}(X, v, t) \rho(v)}{|v|} \mathrm{d} v+\int_{0}^{\infty}|v| \frac{q_{\mathrm{R}}^{2}(X, v, t) \rho(v)}{|v|} \mathrm{d} v \\
=\left\langle q_{\mathrm{L}}^{2}(X, v, t) \rho(v)\right\rangle_{-}+\left\langle q_{\mathrm{R}}^{2}(X, v, t) \rho(v)\right\rangle_{+} .
\end{array}
$$

After substituting (4.2) and (4.3) into the above expressions for $q_{\mathrm{L}}$ and $q_{\mathrm{R}}$ and some manipulations, all changes in $Q$ cancel out and so it stays constant.

When $p_{0}(|v|)$ is strictly decreasing, and hence $\rho(v)>0$, then $Q$ is a norm in the space of functions. Thus, the above theorem implies linear stability.

When $p_{0}(|v|)$ is decreasing, but not strictly, then $\rho(v) \geqslant 0$, but there may be regions where $\rho(v)=0$. They correspond to the intervals where $p_{0}^{\prime}=0$, i.e. where $p_{0}$ is constant. On such intervals, the reflection rules (4.2)-(4.3) for the perturbed density, $p_{\mathrm{L}, \mathrm{R}}$, are trivial:

$$
p_{\mathrm{L}}(X,-v, t)=p_{\mathrm{L}}(X, v, t) \quad \text { and } \quad p_{\mathrm{R}}(X, v, t)=p_{\mathrm{R}}(X,-v, t) .
$$

In this case $p_{\mathrm{L}}$ and $p_{\mathrm{R}}$ cannot grow either. Therefore, we obtain linear stability for all non-increasing $p_{0}(|v|)$. 
Next, we turn to unstable solutions. The stationary solution for an initial density, $p_{0}(|v|)$, satisfying (S3) is linearly unstable if some small perturbations grow exponentially in time, i.e. $\left\|p_{1}(x, v, t)\right\| \sim \Lambda^{t}$ for some $p_{1}(x, v, 0)$ and $\Lambda>1$. This is equivalent to having a positive Lyapunov exponent in the subspace spanned by the function $p_{1}$ and its images. To investigate the existence of such perturbations, we first simplify the collision rules (4.2) and (4.3). Consider the following 'symmetric' and 'antisymmetric' linear combinations of $q_{\mathrm{L}}$ and $q_{\mathrm{R}}$ :

$$
q_{+}(x, v, t)=\frac{q_{\mathrm{L}}(x, v, t)+q_{\mathrm{R}}(1-x,-v, t)}{2}
$$

and

$$
q_{-}(x, v, t)=\frac{q_{\mathrm{L}}(x, v, t)-q_{\mathrm{R}}(1-x,-v, t)}{2} .
$$

They are defined for $x<\frac{1}{2}$. The collision rules (4.2)-(4.3) now take the form

$$
q_{+}(X,-v, t)=q_{+}(X, v, t)
$$

and

$$
q_{-}(X,-v, t)=q_{-}(X, v, t)-2\left\langle q_{-}(X, v, t) \rho(v)\right\rangle_{+} .
$$

Hence, $q_{+}$is simply a periodic function in $t$, and so it cannot grow to infinity or decrease to zero. In other words, it cannot affect the stability or instability of the HEs. The latter is determined by $q_{-}$alone. So we will only consider $q_{-}$and omit '-' for brevity. Our collision rule then reduces to a single equation:

$$
q(X,-v, t)=q(X, v, t)-2\langle q(X, v, t) \rho(v)\rangle_{+} .
$$

Next, we demonstrate, by example, that densities $\rho_{0}(|v|)$ for which the stationary solution (2.11) is unstable do exist.

Example. Let $p_{0}$ be a rectangular function defined by

$$
p_{0}(v)= \begin{cases}1 & \text { if } 0.5<|v|<1 \\ 0 & \text { otherwise }\end{cases}
$$

This $p_{0}(v)$ satisfies (S3) but not (3.1). We will show that the corresponding stationary solution is linearly unstable.

First, the function $h=-p_{0}^{\prime}$ is the sum of two delta functions:

$$
h(v)=-\delta_{0.5}+\delta_{1}
$$

(and symmetrically for $v<0$ ). It is easy to compute $\rho$ directly:

$$
\rho=-\frac{1}{3} \delta_{0.5}+\frac{4}{3} \delta_{1}
$$

Now the reflection rule (4.6) implies:

$$
\begin{aligned}
& q(-1)=-\frac{5}{3} q(1)+\frac{2}{3} q(0.5), \\
& q(-0.5)=-\frac{8}{3} q(1)+\frac{5}{3} q(0.5) .
\end{aligned}
$$

Note that only the values $p(x, \pm 0.5, t)$ and $p(x, \pm 1, t)$ will evolve in a non-trivial way, as specified above, since $h(v)=0$ for all $v \notin\{1,0.5,-0.5,-1\}$.

We now construct a linear subspace of functions $q=q_{\text {- }}$ that stays invariant under the above transformations and in which functions grow exponentially in time (since the $q_{+}$component of the perturbation is irrelevant, we set it to zero). We can simplify the construction further by assuming that at time $t=0$

$$
q(x, \pm 1,0)=u_{1}, \quad q(x, 0.5,0)=u_{2}, \quad q(x,-0.5,0)=u_{3}
$$


with some constants $u_{1}, u_{2}, u_{3}$ (the choice of indices $1,2,3$ is rather arbitrary). We note that the functions $p_{\mathrm{L}, \mathrm{R}}(x, v, 0)$ are now piecewise constant and are completely described by the values $u_{1}, u_{2}, u_{3}$. The space of such perturbations is three dimensional.

It is easy to see that at time $t=1$ the functions $p_{\mathrm{L}, \mathrm{R}}$ will again be constant on the same intervals, and hence they will be described by some other constants $u_{1}^{\prime}, u_{2}^{\prime}, u_{3}^{\prime}$. Our collision rule (4.6) implies that the vectors $\boldsymbol{u}^{\prime}=\left(u_{1}^{\prime}, u_{2}^{\prime}, u_{3}^{\prime}\right)^{T}$ and $\boldsymbol{u}=\left(u_{1}, u_{2}, u_{3}\right)^{T}$ are related by a linear transformation,

$$
\boldsymbol{u}^{\prime}=\boldsymbol{A u}
$$

where $A$ is a $3 \times 3$ matrix:

$$
\boldsymbol{A}=\frac{1}{3}\left(\begin{array}{rrr}
-5 & 2 & 0 \\
0 & 0 & 3 \\
-8 & 5 & 0
\end{array}\right)
$$

After that, the evolution will proceed periodically-the vector $\boldsymbol{u}$ will be multiplied by the matrix $\boldsymbol{A}$ at times $t=1,2,3, \ldots$. The matrix $\boldsymbol{A}$ has three real eigenvalues:

$$
\lambda_{1,2}=\frac{-4 \pm \sqrt{7}}{3} \quad \text { and } \quad \lambda_{3}=1 .
$$

The largest eigenvalue, $\lambda=-(4+\sqrt{7}) / 3 \approx-2.215$, has the following (unit) eigenvector:

$$
\boldsymbol{u}=(0.4472,-0.3680,0.8152) \text {. }
$$

This eigenvector spans a one-dimensional subspace in the space of perturbation densities, which is invariant during time intervals of period 1 and in which the corresponding perturbations are expanded by a factor $|\lambda| \approx 2.215$. Roughly, the perturbations double over one period.

To explore the above periodic growth of perturbations, we note that the piston velocity is given by

$$
\begin{aligned}
V(t) & =\frac{\varepsilon}{2}\left(\left\langle q_{\mathrm{L}}(X, v, t) \rho(v)\right\rangle_{+}-\left\langle q_{\mathrm{R}}(X, v, t) \rho(v)\right\rangle_{-}\right) \\
& =\varepsilon\langle q(X, v, t)\rangle_{+} .
\end{aligned}
$$

Hence in our example, during the time interval $0<t<1$,

$$
V=\frac{\varepsilon}{3}\left(4 u_{1}-u_{3}\right)=0.9736 \varepsilon
$$

During the next time interval, $1<t<2$, we have

$$
V=\frac{\varepsilon}{3}\left(4 u_{1}^{\prime}-u_{3}^{\prime}\right)=-2.156 \varepsilon
$$

and so on. Hence, over a unit period of time, the piston velocity grows by a factor of $|\lambda|=2.215$ and changes sign - the piston starts its movements back and forth (oscillations), which increase exponentially in time.

We note that the same density (4.7) was studied in [CL], where the trajectory of the piston was computed after an initial configuration of gas molecules was selected randomly from the distribution, $p_{0}(v)$, given in (4.7). It was found [CL] that the piston indeed made oscillations that increased exponentially in time. The piston's velocity grew as constant $R^{t}$, with $R \approx 1.6$. This estimate is to be compared with our calculation of the largest eigenvalue $\approx 2.215$.

Next, we modify the unstable perturbations, $q$, found above and make them smooth (rather than piecewise constant) functions of $v$.

We will be looking for the function $q$ of the form

$$
q(x, v, t)=C(v) \mathrm{e}^{z(t-x / v)}
$$


where $z$ is a complex constant. Note that due to (2.2) the function $q$ (with $v$ fixed) can only depend on $t-x / v$. We chose the exponential form in order to investigate the existence of solutions of the linear equation that grow exponentially with time. Also, for convenience, we introduce the new space coordinate, $y$, in the following way: for all $v>0$ and $x<0.5$, we set $y=x+0.5$; for $v<0$ and $x<0.5$, we set $y=0.5-x$; for $v>0$ and $x>0.5$, we set $y=x-0.5$; and for $v<0$ and $x>0.5$, we set $y=1.5-x$. The coordinate $y$ assumes the value zero when a point $(x, v) \in G$ moving under $F^{t}$ reflects off the piston, then grows to 0.5 when the point travels to the wall, and grows further from 0.5 to 1 when the point travels from the wall back to the piston.

In the new coordinate $y$, we will be looking for the function $q$ of the form

$$
q(y, v, t)=C(|v|) \mathrm{e}^{z(t-y /|v|)} .
$$

More precisely, let

$$
\begin{aligned}
& q(y, \pm 1, t)=C(1) \mathrm{e}^{z(t-y)} \\
& q(y, \pm 0.5, t)=C(0.5) \mathrm{e}^{z(t-2 y)} .
\end{aligned}
$$

Recall that $p_{0}(|v|)$ is the characteristic function of the interval $[0.5,1]$.

Now, the reflection rule (4.6) implies

$$
\begin{aligned}
& C(1)=-\frac{5}{3} C(1) \mathrm{e}^{-z}+\frac{2}{3} C(0.5) \mathrm{e}^{-2 z} \\
& C(0.5)=-\frac{8}{3} C(1) \mathrm{e}^{-z}+\frac{5}{3} C(0.5) \mathrm{e}^{-2 z}
\end{aligned}
$$

We need to find $z$ for which the above system of equations has a non-trivial solution. Put $\mathrm{e}^{z}=\lambda$ and introduce an auxiliary variable $D(0.5)=C(0.5) \mathrm{e}^{-z}$. Now the above system can be rewritten as

$$
\begin{aligned}
& \lambda C(1)=-\frac{5}{3} C(1)+\frac{2}{3} D(0.5) \\
& \lambda D(0.5)=C(0.5) \\
& \lambda C(0.5)=-\frac{8}{3} C(1)+\frac{5}{3} D(0.5) .
\end{aligned}
$$

Hence, $\lambda$ is an eigenvalue of the matrix of coefficients

$$
\frac{1}{3}\left(\begin{array}{rrr}
-5 & 2 & 0 \\
0 & 0 & 3 \\
-8 & 5 & 0
\end{array}\right)
$$

which is the same matrix, $\boldsymbol{A}$, that we encountered before. We take its leading eigenvalue $|\lambda|>1$ and set

$$
z=\ln |\lambda|+\mathrm{i} \pi
$$

The function $q$ now takes form

$$
q(y, v, t)= \pm C(|v|)|\lambda|^{t-y /|v|} \cos \pi\left(t-\frac{y}{|v|}\right)
$$

where $C(0.5)$ and $C(1)$ are the coordinates of the leading eigenvector, and we only take the real part, for obvious reasons. Since $|\lambda|>1$, we have an exponential growth of perturbations and thus linear instability. This gives us smooth unstable perturbations.

We now generalize the above construction to arbitrary non-monotonic initial densities $p_{0}$. Let $p_{0}(v)$ satisfy (S3) but not (3.1). We will be looking for perturbations of the form

$$
q(y, v, t)=C(|v|) \mathrm{e}^{z(t-y /|v|)}
$$


with the same convention on $y$ as before. The reflection rule (4.6) leads to (cancelling $\mathrm{e}^{z t}$ )

$$
C(v)=C(v) \mathrm{e}^{-z / v}-2 \int_{0}^{\infty} C(v) \mathrm{e}^{-z / v} \rho(v) \mathrm{d} v
$$

for all $v>0$. Denoting

$$
D=-2 \int C(v) \mathrm{e}^{-z / v} \rho(v) \mathrm{d} v
$$

gives immediately

$$
C(v)=\frac{D}{1-\mathrm{e}^{-z / v}} .
$$

Thus, we not only eliminated $t$ but determined the function $C(v)$ up to a constant factor. The above solution exists if

$$
D=-2 \int_{0}^{\infty} \frac{D \mathrm{e}^{-z / v} \rho(v)}{1-\mathrm{e}^{-z / v}} \mathrm{~d} v
$$

or, cancelling $D$,

$$
\int_{0}^{\infty} \frac{\rho(v)}{1-\mathrm{e}^{z / v}} \mathrm{~d} v=\frac{1}{2} .
$$

If this equation has a solution $z$ with $\operatorname{Re}(z)>0$, we immediately obtain an unstable perturbation (4.8). Otherwise our construction of unstable perturbations does not work.

Unfortunately, it does not seem to be easy to solve equation (4.9) for particular functions $\rho(v)$ or even to determine if it has solutions with a positive real part, as we will demonstrate in the next section.

Next we mention an important property of (4.9). Let us denote

$$
F(z):=\int_{0}^{\infty} \frac{\rho(v)}{1-\mathrm{e}^{z / v}} \mathrm{~d} v-\frac{1}{2} .
$$

Lemma 4.2. $F(z)+F(-z)=0$ for all $z \in \mathbb{C}$.

Proof.

$$
\begin{aligned}
F(z)+F(-z) & =\int_{0}^{\infty} \frac{\rho(v)}{1-\mathrm{e}^{z / v}} \mathrm{~d} v-\int_{0}^{\infty} \frac{\mathrm{e}^{z / v} \rho(v)}{1-\mathrm{e}^{z / v}} \mathrm{~d} v-1 \\
& =\int_{0}^{\infty} \frac{\left(1-\mathrm{e}^{z / v}\right) \rho(v)}{1-\mathrm{e}^{z / v}} \mathrm{~d} v-1 \\
& =0
\end{aligned}
$$

As a result, the existence of a solution of (4.9) with $\operatorname{Re}(z)>0$ is equivalent to that of a solution with $\operatorname{Re}(z)<0$. The alternative is when all the solutions lie on the imaginary axis $\operatorname{Re}(z)=0$.

\section{A special family of densities}

Here, we investigate a family of rectangular densities

$$
p_{0}(v)= \begin{cases}1 & \text { if } r<|v|<1 \\ 0 & \text { otherwise }\end{cases}
$$

where $0<r<1$ is the parameter of our family. Note that our example (4.7) is a particular case of (5.1) with $r=1 / 2$. It is easy to compute

$$
h(v)=-p_{0}^{\prime}(v)=\delta_{r}(v)-\delta_{1}(v)
$$


and

$$
\rho(v)=\frac{v^{2} h(v)}{\int_{0}^{\infty} v^{2} h(v) \mathrm{d} v}=\frac{1}{1-r^{2}}\left[\delta_{1}(v)-r^{2} \delta_{r}(v)\right] .
$$

Since $h(v)=0$ for all $v \notin\{1, r,-1,-r\}$, we only consider perturbations $q(x, v, t)$ defined for $v=1, r,-1,-r$. The reflection rule (4.6) now gives

$$
\begin{aligned}
& q(X,-1, t)=-\alpha q(X, 1, t)+\beta q(X, r, t) \\
& q(X,-r, t)=-\gamma q(X, 1, t)+\alpha q(X, r, t)
\end{aligned}
$$

where

$$
\alpha=\frac{1+r^{2}}{1-r^{2}}, \quad \beta=\frac{2 r^{2}}{1-r^{2}}, \quad \gamma=\frac{2}{1-r^{2}} .
$$

It is relatively easy to investigate the case of rational $r=m / n$ with $1 \leqslant m<n$. Now (5.2) takes form

$$
\alpha=\frac{n^{2}+m^{2}}{n^{2}-m^{2}}, \quad \beta=\frac{2 m^{2}}{n^{2}-m^{2}}, \quad \gamma=\frac{2 n^{2}}{n^{2}-m^{2}} .
$$

To investigate the evolution of perturbations $q(x, v, t)$ as $t$ grows, we consider $n+m$ points $P_{i} \in G, 1 \leqslant i \leqslant n+m$ (shown in figure 1 ). The points $P_{i}$ are defined as follows:

$$
P_{i}= \begin{cases}\left(-1,0.5-\frac{i-1}{m}\right) & \text { for } 1 \leqslant i<\frac{m}{2}+1 \\ \left(1, \frac{i-1}{m}-0.5\right) & \text { for } \frac{m}{2}+1 \leqslant i \leqslant m \\ \left(-r, 0.5-\frac{i-m-1}{n}\right) & \text { for } m<i<m+\frac{n}{2}+1 \\ \left(r, \frac{i-m-1}{n}-0.5\right) & \text { for } m+\frac{n}{2}+1 \leqslant i \leqslant m+n .\end{cases}
$$

It is crucial to observe that the points $P_{i}$ move under the dynamics in a periodic fashion. In a time period $\Delta t=1 / m$, the point $P_{i}$ is mapped to $P_{i+1}$ for all $1 \leqslant i<m$ and all $m+1 \leqslant i<m+n$. Also, $P_{m}$ moves to the piston, gets reflected off it, and lands on $P_{1}$. Likewise, $P_{m+n}$ moves to the piston, gets reflected off it, and lands on $P_{m+1}$. Therefore, the

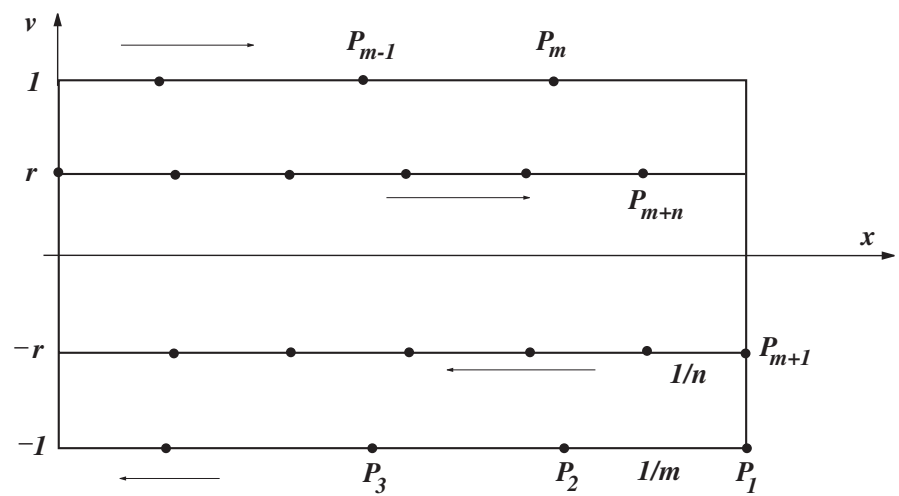

Figure 1. The construction of points $P_{i}$. Here, $m=7$ and $n=12$. 
time shift, $\Delta t$, permutes the points $P_{i}, 1 \leqslant i \leqslant m+n$, in two independent cycles. The reason why we combine the two cycles together is that they are linked by the reflection rule, as we will see shortly.

For each $i$, denote $q_{i}(t)=q\left(P_{i}, t\right)$. Then we have

$$
q_{i}(t+\Delta t)=q_{i-1}(t)
$$

for all $2 \leqslant i \leqslant m$ and $m+2 \leqslant i \leqslant m+n$. The reflection rule now implies

$$
\begin{aligned}
& q_{1}(t+\Delta t)=-\alpha q_{m}(t)+\beta q_{m+n}(t) \\
& q_{m+1}(t+\Delta t)=-\gamma q_{m}(t)+\alpha q_{m+n}(t) .
\end{aligned}
$$

Thus, the vector $\boldsymbol{q}(t)=\left(q_{1}(t), \ldots, q_{n+m}(t)\right)$ is updated at time $t+\Delta t$ by the rule

$$
\boldsymbol{q}(t+\Delta t)=\boldsymbol{B} \boldsymbol{q}(t)
$$

where $\boldsymbol{B}$ is an $(n+m) \times(n+m)$ matrix,

$$
\boldsymbol{B}=\left(\begin{array}{cccccccc}
0 & \cdots & & -\alpha & & \cdots & & \beta \\
1 & \ddots & & & & & & \\
\vdots & \ddots & 0 & \vdots & \vdots & & & \vdots \\
& & 1 & 0 & & \cdots & & \\
0 & \cdots & & -\gamma & 0 & \cdots & & \alpha \\
& & & & 1 & 0 & & \\
\vdots & & & \vdots & & \ddots & \ddots & \vdots \\
0 & \cdots & & 0 & & \cdots & 1 & 0
\end{array}\right) .
$$

We conclude that the existence of unstable perturbations $q(t)$ is equivalent to the existence of an eigenvalue $\lambda$ of $\boldsymbol{B}$ such that $|\lambda|>1$. The characteristic polynomial of the matrix $\boldsymbol{B}$ is

$$
P(\lambda)=\lambda^{m+n}+\alpha \lambda^{n}-\alpha \lambda^{m}-1
$$

where $\alpha=\left(n^{2}+m^{2}\right) /\left(n^{2}-m^{2}\right)$ is defined in (5.3).

Remark. Interestingly, the equation (4.9) can be reduced to $P(\lambda)=0$ as well. Indeed, it is easy to see that

$$
\int_{0}^{\infty} \frac{\rho(v)}{1-\mathrm{e}^{z / v}} \mathrm{~d} v=\frac{1}{1-r^{2}}\left[\frac{1}{1-\mathrm{e}^{z}}-\frac{r^{2}}{1-\mathrm{e}^{z / r}}\right] .
$$

Now the substitution $\lambda=\mathrm{e}^{z / m}$ and some algebraic manipulations show that equation (4.9) is equivalent to $P(\lambda)=0$.

It is easy to see that if $\lambda$ is a root of $P(\lambda)$, then so is $1 / \lambda$ (this reciprocability also follows from lemma 4.2). Thus, the existence of unstable perturbations is equivalent to the existence of eigenvalues of $\boldsymbol{B}$ that do not lie on the unit circle $|\lambda|=1$.

If an eigenvalue $|\lambda|>1$ of $\boldsymbol{B}$ exists, then the perturbations in the corresponding eigenspace grow by a factor of $|\lambda|$ over the time period $\Delta t=1 / \mathrm{m}$. Hence, the expansion factor per unit time would be $\Lambda=|\lambda|^{m}$.

Theorem 5.1. Let $r=m / n$ be a rational number with an even denominator $n$ (hence, $m$ is odd). Then there is a unique eigenvalue of $\boldsymbol{B}$ such that $\lambda<-1$. This eigenvalue has multiplicity 1. The expansion factor per unit time $\Lambda_{r}=|\lambda|^{m}$ depends on $r$ continuously, and we have, asymptotically,

$$
\Lambda_{r}=1+\text { const } \cdot r^{3 / 2}+\mathcal{O}\left(r^{2}\right) \quad \text { as } r \rightarrow 0
$$


and

$$
\Lambda_{r} \sim \frac{\text { const }}{1-r} \quad \text { as } r \rightarrow 1
$$

Proof. One can easily check that, under the conditions of the theorem, $P(-1)>0$ and $P(-\infty)<0$, and hence a root $\lambda<-1$ exists. Next,

$$
P^{\prime}(\lambda)=\left[(n+m) \lambda^{n}+\alpha n \lambda^{n-m}-\alpha m\right] \lambda^{m-1}
$$

and so $P^{\prime}(-1)<0$ and $P^{\prime}(-\infty)>0$. Now let $Q(\lambda)=(n+m) \lambda^{n}+\alpha n \lambda^{n-m}-\alpha m$; then

$$
Q^{\prime}(\lambda)=\left[(n+m) \lambda^{m}+\alpha(n-m)\right] n \lambda^{n-m-1}
$$

and so clearly $Q^{\prime}(\lambda)<0$ for all $\lambda<1$. Putting these facts together proves the uniqueness and the simplicity of the root $\lambda<0$.

The equation $P(\lambda)=0$ can be rewritten in terms of $\Lambda_{r}=|\lambda|^{m}$ as follows:

$$
-\Lambda_{r}^{1+1 / r}+\frac{1+r^{2}}{1-r^{2}} \Lambda_{r}^{1 / r}+\frac{1+r^{2}}{1-r^{2}} \Lambda_{r}-1=0 .
$$

Now the continuity of $\Lambda_{r}$, as a function of $r$, is obvious. Note that our argument is only valid when $r=m / n$ with an even $n$ and an odd $m$ because this parity condition dictates the signs in (5.7).

To prove (5.5), one can substitute $\Lambda_{r}=1+\varepsilon$ into (5.7) and expand all the terms in Taylor series, the calculation is then straightforward and we omit it. The proof of (5.6) is similar.

Figure 2 presents the graph of the Lyapunov exponent, $\log \Lambda_{r}$ as a function of $r$.

Lemma 5.2. Let $z$ be a solution of (4.9) such that $\left|\mathrm{e}^{z}\right| \neq 1$ and $\mathrm{e}^{z} \in \mathbb{R}$. Then $\mathrm{d} F / \mathrm{d} z \neq 0$ (in fact, $\mathrm{d} F / \mathrm{d} z$ is a real negative number).

Proof. A direct calculation shows that

$$
\frac{\mathrm{d} F}{\mathrm{~d} z}=-\frac{\left(1+r+r^{2}\right)\left(1+\mathrm{e}^{z}\right)^{2}+r^{3}\left(1-\mathrm{e}^{z}\right)^{2}}{4 r^{2}(1+r)\left(1-\mathrm{e}^{z}\right)^{2}}
$$

which proves the lemma.

For any $r=m / n$ with even $n$ and odd $m$, the corresponding solution, $\mathrm{e}^{z}=-\Lambda_{r}$, satisfies the conditions of the above lemma. Hence, this solution changes continuously with $r$, and so we get the following corollary.

Corollary 5.3. For every $r=m / n$ with even $n$ and odd $m$, there is an interval $(r-\varepsilon, r+\varepsilon)$ in which all parameter values have unstable perturbations.

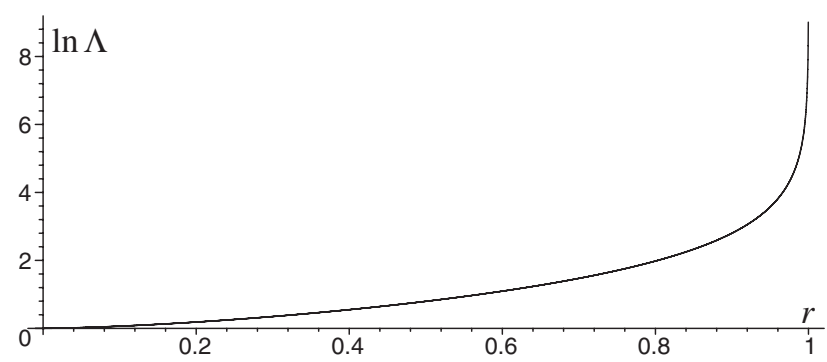

Figure 2. $\log \Lambda_{r}$ as a function of $r$. 
Therefore, unstable perturbations exist for an open and dense set of parameter values $0<r<1$. Moreover, one can show [C] that equation (4.9) has solutions with $\operatorname{Re}(z) \neq 0$, implying the existence of unstable perturbations, for all irrational $r \in(0,1)$. One would naturally wonder if all $r$ have unstable perturbations. The answer is, surprisingly, negative.

Fact 5.4. For the density (5.1) with $r=1 / 3$, there are no solutions, $z$, of (4.9) with $\operatorname{Re}(z)>0$, and hence there are no solutions of the linearized equation that grows exponentially with time.

Proof. The characteristic equation,

$$
\lambda^{4}+\frac{5}{4} \lambda^{3}-\frac{5}{4} \lambda-1=0
$$

has two real roots $(\lambda= \pm 1)$ and two complex roots. The complex roots are, on the one hand, conjugate and, on the other, satisfy the reciprocability rule, $P(\lambda)=0$, if and only if $P(1 / \lambda)=0$. Hence, they must belong to the unit circle $|\lambda|=1$.

It is interesting to know if other rational parameter values $r=m / n$ with odd $n$ are also stable. We have examined the values $r=1 / n$ for small odd values of $n=5,7, \ldots, 31$ numerically (using MATLAB) and always found that all the roots of $P(\lambda)$ belonged to the unit circle. Therefore, we conjecture that the values $r=1 / n$ with odd $n$ are stable.

On the other hand, the values $r=m / n$ with odd $n$ but $m>1$ appear to be unstable. For $r=2 / 3,2 / 5,3 / 5,3 / 7$ we found, again numerically (using MATLAB), roots $\lambda$ such that $|\lambda|>1$. All those roots are complex; for example, for $r=2 / 3$ they are $\lambda=-0.3778 \pm 1.7173 \mathrm{i}$. It remains for us to determine theoretically whether all rational values $r=m / n$ with $m>1$ are unstable, and we leave this question open.

Fact 5.4 seems to disagree with theorem 5.1. Indeed, let $p_{0}(v)$ be the rectangular density (5.1) corresponding to $r=1 / 3$ and $p(x, v, 0)=p_{0}(v)+\varepsilon p_{1}(x, v, 0)$ an arbitrary perturbation with an infinitesimally small $\varepsilon$. According to fact 5.4 , this perturbation cannot grow exponentially in time. On the other hand, let us approximate $1 / 3$ by a rational number $r=m / n$ with even $n$. Denote by $p_{0}^{*}(v)$ the corresponding rectangular density (5.1) for the chosen $r=m / n$. Then we have

$$
p(x, v, 0)=p_{0}^{*}(v)+\varepsilon p_{2}(x, v, 0) \quad \text { with } p_{2}=p_{1}+\frac{p_{0}-p_{0}^{*}}{\varepsilon} .
$$

Hence, if $|r-1 / 3|<\varepsilon$, then $\left(p_{0}-p_{0}^{*}\right) / \varepsilon$ is of order one (in the $L^{1}$ metric), and $p(x, v, 0)$ becomes an $\varepsilon$-perturbation of the density, $p_{0}^{*}(v)$. As such, it 'must' grow exponentially in time according to theorem 5.1. This apparent disagreement requires an explanation, which we provide next.

We recall that smooth unstable perturbations are given by the general formula (4.8). For the rectangular density (5.1), the velocity $v$ in this formula only takes two values, $|v|=r$ and $|v|=1$; hence the factor $C(|v|)$ takes two values as well and so plays only a limited role. For simplicity, we set $|v|=1$ and ignore the constant factor $C(|v|)=C(1)$. Now the (real part of) unstable perturbations is described by

$$
q(y, 1, t)=\operatorname{Re} \mathrm{e}^{z(t-y)}=\mathrm{e}^{(\operatorname{Re} z)(t-y)} \cos [(\operatorname{Im} z)(t-y)] .
$$

A similar formula holds for $|v|=r$, and we omit it. Now recall that for any rational $r=m / n$ we have $\mathrm{e}^{z / m}=\lambda$, where $\lambda<-1$ is the eigenvalue of $\boldsymbol{B}$, described by theorem 5.1. Therefore, $\operatorname{Re} z=m \log |\lambda|=\log \Lambda$ and $\operatorname{Im} z= \pm m \pi$.

We see that the real part of $z$ changes continuously with $r=m / n$ but the imaginary part does not. In particular, when $r=m / n$ is close to $1 / 3$ and $n$ is even, both $m$ and $n$ have to be large, so that $m \rightarrow \infty$ and $|\operatorname{Im} z| \rightarrow \infty$ as $r \rightarrow 1 / 3$. In terms of the perturbation (5.9), the growth of $|\operatorname{Im} z|$, as $r$ approaches $1 / 3$, implies that the function $q(y, 1, t)$ 


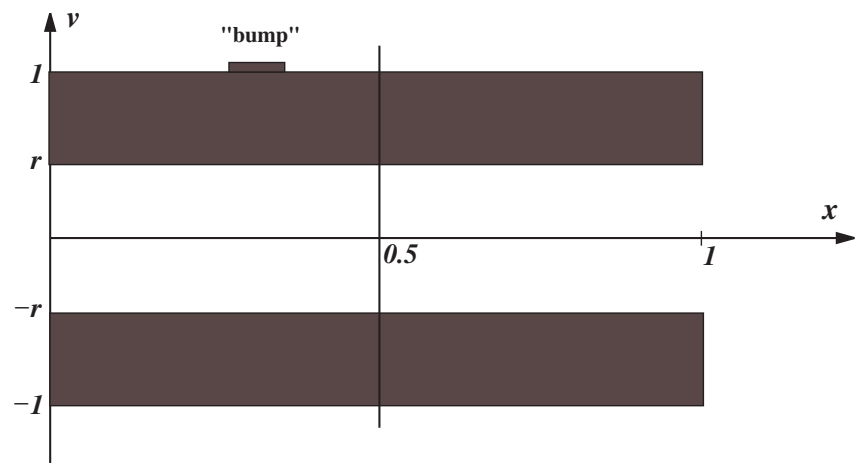

Figure 3. Initial rectangular density (5.1) perturbed by a 'bump'.

becomes highly oscillatory, and so does the corresponding initial unstable perturbation $p(x, v, 0)=h(v) q(x, v, 0)$. Thus, the linear subspace of unstable perturbations (along which exponential growth takes place) becomes nearly orthogonal to any given function, in particular to $p_{2}(x, v, 0)$, defined in $(5.8)$.

This explains the above 'disagreement'. The density, $p_{2}$, does grow exponentially in time for any $r=m / n$ with an even $n$, but as $r \rightarrow 1 / 3$, the projection of $p_{2}$ onto the unstable subspace corresponding to the positive Lyapunov exponent $\log \Lambda_{r}>0$ becomes small and vanishes in the limit, and hence the exponential growth is not visible during a long initial interval of time. In the limit $r \rightarrow 1 / 3$, that 'initial interval' becomes infinite and the instability evaporates.

One can also reverse this line of argument. Indeed, when $\varepsilon$ is not infinitesimally small but finite, the representation (5.8) implies that any perturbation $p(x, v, 0)$ of the rectangular density (5.1) for any $0<r<1$ will eventually grow exponentially fast in time (because any $r \in(0,1)$ can be approximated by rational numbers $m / n$ with even $n)$. We checked this conclusion experimentally and found that it was indeed correct.

To investigate the instability experimentally, we solved the HEs (H1)-(H4) numerically, starting with a perturbed rectangular density (5.1), shown in figure 3. The initial density, $p(x, v, 0)$, takes the value 1 on the black region and 0 elsewhere. The small 'bump' at the top left edge of the upper rectangle represents the perturbation. The area of the bump in our experiments was less than $1 \%$ relative to the total area of each black rectangle.

Figures 4 and 5 show typical trajectories of the piston, $X(t)$, for $r=1 / 3$ and $r=1 / 4$, respectively. Note that their shape during the first 4-5 units of time only reflects the size and position of the 'bump'; hence the shape is almost identical. For times $\tau>5$, though, interesting differences develop. In the $r=1 / 4$ unstable case, the oscillations grow exponentially in a pronounced steady fashion. In the $r=1 / 3$ linearly stable case, the oscillations distort their shape first, then start growing slowly, and only pick up pace at $\tau \sim 12$. Eventually, they also grow exponentially. These observations indicate that the linearly stable stationary solution with $r=1 / 3$ is nonlinearly (globally) unstable.

\section{Periodic solutions of the HEs}

Here we discuss the long-term behaviour of our system in the unstable regime.

In our previous work [CL] we reported the results of computer simulations of the piston and particle dynamics in an ideal gas with many (up to 27 million) particles. The initial 


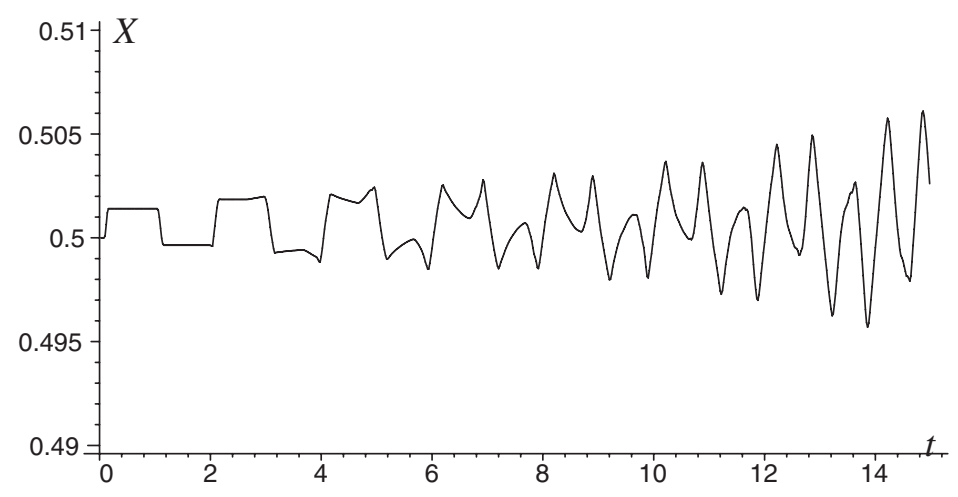

Figure 4. Piston's trajectory from the solution of the HE for a perturbed rectangular density with $r=1 / 3$.

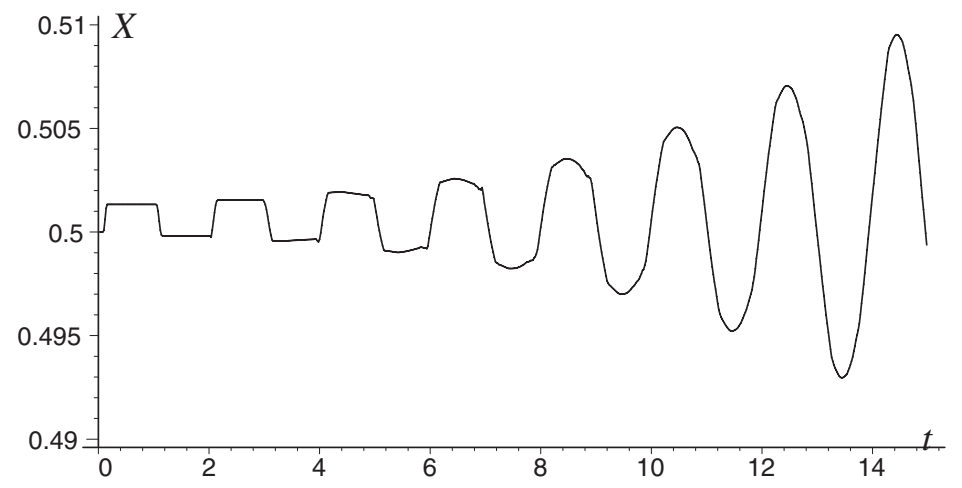

Figure 5. Piston's trajectory from the solution of the HE for a perturbed rectangular density with $r=1 / 4$.

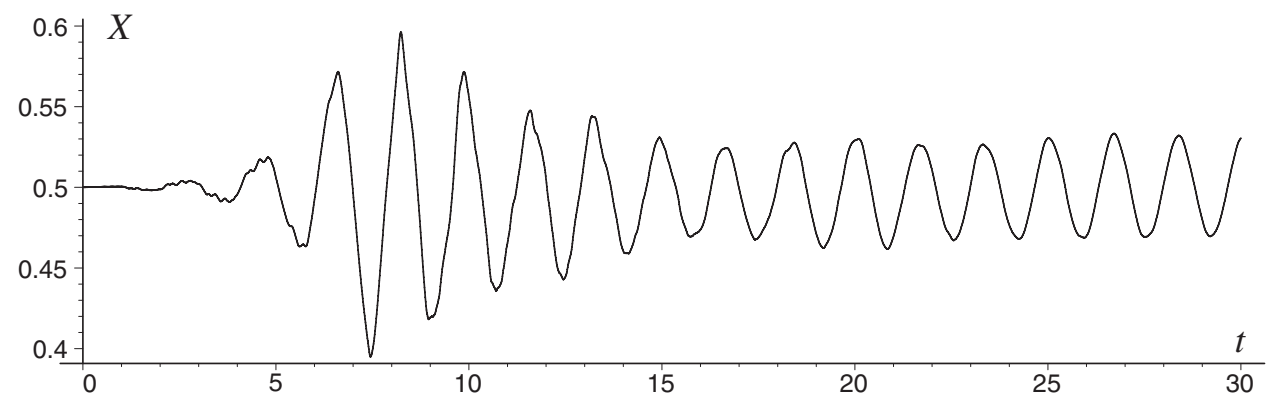

Figure 6. Piston's trajectory in the mechanical model with $10^{6}$ particles.

configuration of particles was selected randomly with the average density (4.7) (see [CL] for details). A typical trajectory of the piston, $X(t)$, found in our experiments is shown here in figure 6. One can see that during the initial interval of time, $0<t<8$, the piston moves back and forth with an exponentially increasing amplitude, which is consistent with our analysis in section 4 , where the density (4.7) was proven to be unstable.

Later on, however, at times $8<t<15$, the amplitude of the piston's oscillations decreases to a certain constant value (nearly a half of its maximum, attained at $t=8$ ). Then the piston's 


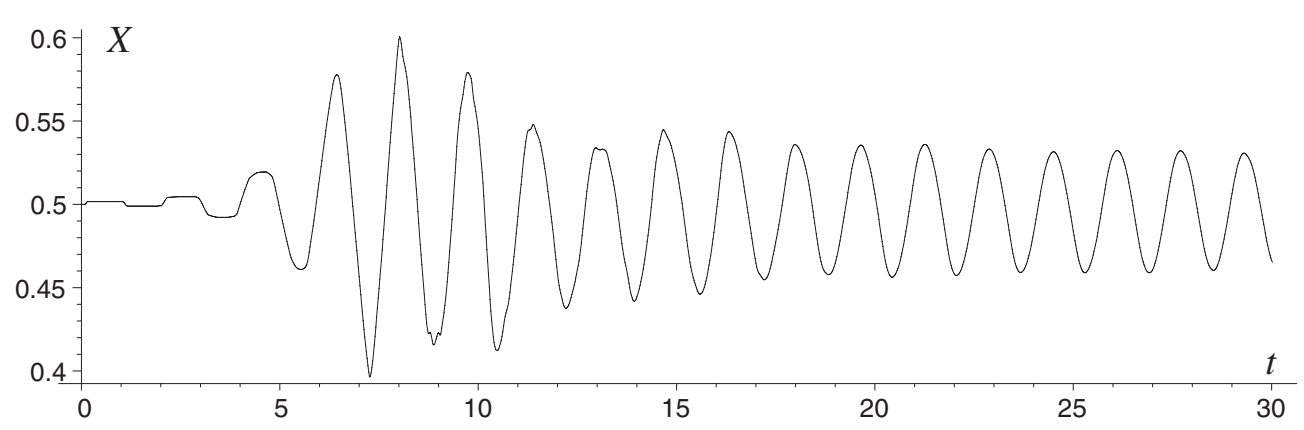

Figure 7. Piston's trajectory from the HE for a perturbed rectangular density with $r=1 / 2$.

oscillations become very stable and continue almost unchanged for a very long time, up to $t=50$ or 100 , with a very slowly decreasing amplitude.

On the other hand, we have solved the HEs (H1)-(H4) numerically, starting with the same initial density (4.7), perturbed by a bump (shown in figure 3). Figure 7 presents the resulting trajectory of the piston. One can see that it behaves almost identically to the simulated trajectory of the piston shown in figure 6 . Thus, not only the initial instability but also the longer term behaviour of the simulated piston trajectory approximately match those of perturbed solutions of the HEs.

The behaviour shown in figure 7 persists when various perturbations of the initial density (4.7) are applied. It seems that there is a periodic cycle or an invariant manifold of quasi-periodic solutions of $(\mathrm{H} 1)-(\mathrm{H} 4)$ that acts as an attractor. Of course, due to the time-reversibility of the HEs there can be no attractors in the strict sense. It is more likely that there is an invariant manifold of periodic or quasi-periodic solutions that acts as a saddle point in the phase space: typical trajectories approach that manifold temporarily and then slowly move away. We cannot prove rigorously the existence of periodic or quasi-periodic solutions, but we construct such solutions by using perturbative analysis.

We will be looking for solution of the HEs (H1)-(H4) such that the piston makes harmonic oscillations

$$
X(t)=\frac{1}{2}+\varepsilon \cos \omega t, \quad \dot{X}(t)=-\varepsilon \omega \sin \omega t
$$

with some fixed $\omega>0$ and small $\varepsilon>0$. We will approximate such solutions up to the first order in $\varepsilon$, i.e. ignoring terms of higher order.

The construction is done in two steps. First, we assume that the piston moves as prescribed by (6.1) and consider the motion of a fluid point bouncing against the moving piston, $X(t)$, and the fixed wall, $x=0$. Second, we define the density, $p(x, v, t)$, which, coupled with the piston's oscillations (6.1), satisfies equations $(\mathrm{H} 1)-(\mathrm{H} 4)$.

Let the piston move according to equations (6.1). Then fluid points in the left compartment, $0<x<X(t)$, bounce against the wall, $x=0$, and the piston; the latter simply acts on them as a moving wall. It is known that the phase space of gas particles bouncing against a periodically moving wall necessarily contains many invariant curves. Moreover, the region corresponding to high velocities $|v|>v_{0}$ is densely filled by such invariant curves; the larger $v_{0}$, the higher the density of invariant curves. This fact is a consequence of KAM theory, and it was first proved by Douady in his thesis [Do] and later independently by Laederich and Levi [LL]. We describe these invariant curves approximately, up to the first order in $\varepsilon$, by equation

$$
v+\varepsilon F(t, V)=V+\mathcal{O}\left(\varepsilon^{2}\right)
$$


where $v$ denotes the velocity of the particle when it kicks the piston, $t$ is the collision time, and $V$ is the parameter of the curve. In fact, we will construct invariant curves for all $V>V_{0}$ with some $V_{0}>0$. Here, we only consider particles to the left to the piston; the particles to the right of the piston are completely symmetric.

Let us consider successive collisions of a gas particle with the piston. Denote by $v_{n}>0$ the velocity of the particle before its $n$th collision and by $t_{n}$ the time of that collision. Then the law of elastic impact reads

$$
v_{n+1}=v_{n}-2 \dot{X}\left(t_{n}\right)=v_{n}+2 \varepsilon \omega \sin \omega t_{n} .
$$

Let $t_{n+1 / 2}$ denote the time at which the particle bounces off the wall, $x=0$, between its $n$th and $(n+1)$ th collisions with the piston. Obviously, $t_{n+1 / 2}=t_{n}+X\left(t_{n}\right) / v_{n+1}$ and $v_{n+1}\left(t_{n+1}-t_{n+1 / 2}\right)=X\left(t_{n+1}\right)$. Since we are interested in knowing $v_{n}$ up to terms $\mathcal{O}(\varepsilon)$, it is sufficient to find $t_{n}$ up to terms $\mathcal{O}(1)$. This is easy:

$$
t_{n+1}=t_{n}+\frac{1}{v_{n+1}}+\mathcal{O}(\varepsilon)=t_{n}+\frac{1}{V}+\mathcal{O}(\varepsilon)
$$

where we used (6.2).

Now let us look for an invariant curve of the form

$$
v+\varepsilon F(t, V)=V+\mathcal{O}\left(\varepsilon^{2}\right) .
$$

We have to impose the constraint

$$
v_{n+1}+\varepsilon F\left(t_{n+1}, V\right)=v_{n}+\varepsilon F\left(t_{n}, V\right)+\mathcal{O}\left(\varepsilon^{2}\right) .
$$

By equations (6.3), (6.4), and (6.5), we get

$$
v_{n}+2 \varepsilon \omega \sin \omega t_{n}+\varepsilon F\left(t_{n}+\frac{1}{V}+\mathcal{O}(\varepsilon), V\right)=v_{n}+\varepsilon F\left(t_{n}, V\right) .
$$

Cancelling $v_{n}$ and $\varepsilon$ and removing the index $n$ gives a general equation for an invariant curve:

$$
2 \omega \sin \omega t+F\left(t+\frac{1}{V}, V\right)-F(t, V)=0 .
$$

We construct solutions of this equation in the form

$$
F(t, V)=a \cos \omega t+b \sin \omega t
$$

where $a$ and $b$ depend on $V$. By substituting this expression into (6.6), we find that (6.6) can only hold if

$$
\begin{aligned}
& a\left(\cos \left(\frac{\omega}{V}\right)-1\right)+b \sin \left(\frac{\omega}{V}\right)=0 \\
& a \sin \left(\frac{\omega}{V}\right)-b\left(\cos \left(\frac{\omega}{V}\right)-1\right)=2 \omega .
\end{aligned}
$$

The solution of the above system is

$$
\begin{aligned}
& a=\frac{\omega \sin (\omega / V)}{1-\cos (\omega / V)} \\
& b=\omega .
\end{aligned}
$$

Remark. Note that $a$ (and hence the invariant curve) is not defined for $V=\omega / 2 \pi k$, $k= \pm 1, \pm 2, \ldots$. To avoid these singularities, we will not use invariant curves corresponding to $V \leqslant \omega / 2 \pi$. In particular, the density $p(x, v, t)$ that we define below will be constant for $|v| \leqslant \omega / 2 \pi$. 
Thus, for any $V>V_{0}>\omega / 2 \pi$, we can define an invariant curve $u(x, t ; V)$ in the phase space of gas particles, where $V$ is the parameter of the curve and $u(x, t ; V)$ is the velocity of the particle on the curve at point $x$ at time $t$. The curve is made by two branches: the upper branch, $u^{+}$, and the lower branch, $u^{-}$. Obviously, we have

$$
\begin{aligned}
& u^{+}(X(t), t ; V)=V-\varepsilon F(t, V)+\mathcal{O}\left(\varepsilon^{2}\right) \\
& u^{-}(X(t), t ; V)=-[V-\varepsilon F(t, V)-2 \dot{X}(t)]+\mathcal{O}\left(\varepsilon^{2}\right) \\
& u^{+}(t, 0, V)=-u^{-}(t, 0, V) .
\end{aligned}
$$

Note that the last equation here is equivalent to (6.5).

Now, we define a density $p(x, v, t)$ so that its value on each invariant curve $u(x, t ; V)$, $|V|>V_{0}$, is a constant denoted by $\rho(V)$. Between the curves $u^{+}\left(x, t ; V_{0}\right)$ and $u^{-}\left(x, t ; V_{0}\right)$, we set the density to a constant equal to 1 . Therefore,

$$
p\left(x, u^{+}(t, x, V), t\right)=p\left(x, u^{-}(t, x, V), t\right)=\rho(V) \quad \text { if } V>V_{0}
$$

and

$$
p(x, v, t) \equiv 1 \quad \text { if } u^{-}\left(x, t ; V_{0}\right)<v<u^{+}\left(x, t ; V_{0}\right)
$$

The function $\rho(V)$ and the 'cutoff' value $V_{0}>\omega / 2 \pi$ will be specified below.

Example. Let us set $\rho(V) \equiv 0$ for $V>V_{0}$, i.e.

$$
p(x, v, t)= \begin{cases}1 & \text { for } u^{-}\left(x, t ; V_{0}\right)<u<u^{+}\left(x, t ; V_{0}\right) \\ 0 & \text { elsewhere. }\end{cases}
$$

In order to compute the pressure on the piston, we only need to know the density, $p(x, v, t)$, at the point $x=X(t)$, i.e. we need to know the function

$$
v(t, V):=u^{+}(X(t), t, V)=V+\varepsilon F(t, V)+\mathcal{O}\left(\varepsilon^{2}\right) .
$$

In our example the density on the piston (on the left-hand side) is 1 up to $v^{+}=V_{0}-\varepsilon F\left(V_{0}, t\right)$. The density on the piston on the right-hand side is 1 up to a similar invariant curve, which is phase shifted by $\Delta t=\pi / \omega$. Therefore, the density on the right-hand side is 1 down to $v^{-}=-V_{0}-\varepsilon F\left(t, V_{0}\right)$. Since $F(t+\pi / \omega)=-F(t)$ by (6.7), the velocity of the piston is exactly the average of $v^{+}$and $v^{-}$and therefore is $-\varepsilon F\left(t, V_{0}\right)$.

Thus, our density and the piston satisfy the HEs (H1)-(H4) if $\dot{X}=-\varepsilon F\left(t, V_{0}\right)$, which gives

$$
-\varepsilon \omega \sin \omega t=-\varepsilon \omega \sin \omega t-\frac{\varepsilon \omega \sin \left(\omega / V_{0}\right) \cos \omega t}{1-\cos \left(\omega / V_{0}\right)} .
$$

In our example, the only possible choice is $V_{0}=\omega / \pi$.

Now let us consider the case of a generic function $\rho(V)$. The pressure on the piston on the left-hand side is equal to

$$
P_{\mathrm{L}}=\int_{\dot{X}}^{\infty} p_{\mathrm{L}}(v)(v-\dot{X})^{2} \mathrm{~d} v=\int_{0}^{\infty} p_{\mathrm{L}}(v)\left(v^{2}-2 v \dot{X}\right) \mathrm{d} v+\mathcal{O}\left(\varepsilon^{2}\right)
$$

where $p_{\mathrm{L}}(v)=p(X(t)-0, v, t)$ is the density on the piston (we have used the fact that $\dot{X}=\mathcal{O}(\varepsilon))$. Recall that the density is $p_{\mathrm{L}}(v)=\rho(V)=\rho(v+\varepsilon F(t, v))+\mathcal{O}\left(\varepsilon^{2}\right)$. From now on we neglect terms of order $\mathcal{O}\left(\varepsilon^{2}\right)$. Then we get

$$
P_{\mathrm{L}}=\int_{0}^{\infty}\left(v^{2}-2 v \dot{X}\right) \rho(v+\varepsilon F(t, v)) \mathrm{d} v .
$$


The pressure on the right-hand side is given, by analogy, by

$$
P_{\mathrm{R}}=\int_{-\infty}^{0} p_{\mathrm{R}}(v)\left(v^{2}-2 v \dot{X}\right) \mathrm{d} v=\int_{0}^{\infty} p_{\mathrm{R}}(-v)\left(v^{2}+2 v \dot{X}\right) \mathrm{d} v .
$$

Note that for $v>0$ we have $p_{\mathrm{R}}(v)=\rho(V)=\rho(v+\varepsilon F(v, t+\pi))=\rho(v-\varepsilon F(t, v))$. Therefore,

$$
P_{\mathrm{R}}=\int_{0}^{\infty}\left(v^{2}+2 v \dot{X}\right) \rho(v-\varepsilon F(t, v)) \mathrm{d} v .
$$

We now conclude that $P_{\mathrm{L}}=P_{\mathrm{R}}$ iff

$$
\dot{X}=\varepsilon \frac{\int_{0}^{\infty} \rho^{\prime}(v) F(t, v) v^{2} \mathrm{~d} v}{\int_{0}^{\infty} \rho(v) 2 v \mathrm{~d} v}=-\varepsilon \frac{\int_{0}^{\infty} \rho^{\prime}(v) F(t, v) v^{2} \mathrm{~d} v}{\int_{0}^{\infty} \rho^{\prime}(v) v^{2} \mathrm{~d} v}
$$

which is analogous to our earlier formula (4.1).

Using (6.7) and the subsequent equations, we find

$$
\dot{X}=-\varepsilon \omega \sin \omega t-\varepsilon \frac{\int_{0}^{\infty} \rho^{\prime}(v)(\sin (\omega / v) /(1-\cos (\omega / v))) v^{2} \mathrm{~d} v}{\int_{0}^{\infty} \rho^{\prime}(v) v^{2} \mathrm{~d} v} \omega \cos \omega t
$$

Our density, coupled with the piston oscillations (6.1), satisfies the HEs (H1)-(H4) if and only if $\dot{X}=-\varepsilon \omega \sin \omega t$. This implies

$$
\int_{\omega / 2 \pi}^{\infty} \mathrm{d} v \rho^{\prime}(v) v^{2} \frac{\sin (\omega / v)}{1-\cos (\omega / v)}=0
$$

where we have imposed $\rho^{\prime}=0$ for $v \leqslant \omega / 2 \pi$.

Interestingly, (6.8) is related to our early equation (4.9). Specifically, let $z$ in (4.9) be a purely imaginary number, $z=\omega$ i. Also note that $\rho(v)$ in (4.9) is just proportional to $v^{2} \rho^{\prime}(v)$ here. Then (6.8) becomes equivalent to $\operatorname{Im} F(z)=0$, with $F(z)$ defined by (4.10). In other words, (6.8) expresses the 'imaginary part' of the equation (4.9). We already observed in the previous section that $\operatorname{Im} z$ characterized the frequency of oscillations of unstable perturbations, and here $\omega=\operatorname{Im} z$ is the frequency of oscillations of the piston. We note that for $z=\omega \mathrm{i}$ one always has $\operatorname{Re} F(z)=0$, as follows from (4.10), and hence in our case (6.8) is equivalent to $F(z)=0$. Therefore solving (6.8) corresponds to finding pure imaginary eigenvalues of the HE linearized around a stationary solution $\rho$.

This analysis is only relevant when $\rho(v)$ is stable; otherwise the motion will be dominated by other exponentially increasing modes, and so from now on we restrict ourselves to the case in which $\rho$ is a non-increasing function of $|v|$.

In this case, there are some quantitative restrictions on the period of oscillations of the piston. The period $T=2 \pi / \omega$ can be bounded from below by a function of the average kinetic energy $\langle\mathcal{K}\rangle=\mathcal{K} / \mathcal{M}$, where

$$
\mathcal{K}=\int_{0}^{\infty} \rho(v) \frac{v^{2}}{2} \mathrm{~d} v \quad \text { and } \quad \mathcal{M}=\int_{0}^{\infty} \rho(v) \mathrm{d} v
$$

Proposition 6.1. Let $\rho^{\prime} \leqslant 0$ be supported on the interval $[\omega / 2 \pi, \infty)$ and satisfy equation (6.8). Then the period of oscillations, $T$, is bounded by

$$
T \geqslant \sqrt{\frac{2}{3\langle\mathcal{K}\rangle}} .
$$

The equality holds when $\rho^{\prime}=(\pi / \omega) \delta(v-\omega / \pi)$, i.e. when $\rho(|v|)$ is constant on $(0, \omega / \pi)$ and 0 elsewhere. 
Note that equation (6.8) can be satisfied in many different ways, all of them leading to different periodic solutions of the HEs, but it is not clear which of them, if any, describe the long-term behaviour of the system for a given initial condition.

Nevertheless, it is possible to verify proposition 6.1 numerically. For instance, in the case of our unstable density (4.7), we have $\mathcal{K}=\frac{7}{24}$ and $T=\sqrt{16 / 7} \simeq 1.51186$. The experimentally determined period of oscillations of the piston is $T \simeq 1.62$ (see [CL]). We also simulated the piston trajectory with other unstable densities (5.1) with $r \rightarrow 0$ and observed that the period of oscillations approached 2 , which is exactly the value of the lower bound given by the above proposition (because in this case $\mathcal{K}=\frac{1}{6}$ and hence $T=2$ ). The fact that the period converges to 2 as $r \rightarrow 0$ also follows from our early example (with $V_{0}=1$ ), in which $\omega=\pi V_{0}=\pi$; hence $T=2 \pi / \omega=2$.

Proof of proposition 6.1. Consider a function

$$
G(v):=v \frac{\sin (\omega / v)}{1-\cos (\omega / v)}
$$

Then equation $(6.8)$ reads

$$
\mathcal{C}:=\int_{\omega / 2 \pi}^{\infty}-\rho^{\prime} v G \mathrm{~d} v=0 .
$$

Note that in the interval $[\omega / 2 \pi, \infty)$ the function $G(v)$ is strictly increasing and that $G(\omega / 2 \pi)=-\infty, G(\omega / \pi)=0$, and $G(\infty)=\infty$.

Introducing a new function $R(v)=-\rho^{\prime}(v) v \geqslant 0$ and integrating by parts yields

$\mathcal{M}=\int_{\omega / 2 \pi}^{\infty} R(v) \mathrm{d} v, \quad \mathcal{K}=\int_{\omega / 2 \pi}^{\infty} R(v) \frac{v^{2}}{6} \mathrm{~d} v, \quad \mathcal{C}=\int_{\omega / 2 \pi}^{\infty} R(v) G(v) \mathrm{d} v$.

It is useful to replace $v$ by a new variable $u=G(v),-\infty<u<\infty$. Since $G$ is strictly increasing, we can write

$$
\mathcal{M}=\int_{-\infty}^{\infty} S(u) \mathrm{d} u, \quad \mathcal{K}=\int_{-\infty}^{\infty} S(u) \eta(u) \mathrm{d} u, \quad \mathcal{C}=\int_{\infty}^{\infty} S(u) u \mathrm{~d} u
$$

where $S(u)=R\left(G^{-1}(u)\right) / G^{\prime}\left(G^{-1}(u)\right)$ and $\eta(u)=\left(G^{-1}(u)\right)^{2} / 6$ (here $G^{-1}$ denotes the inverse of the function $G)$.

We have to solve the following variational problem: minimize $\mathcal{K} / \mathcal{M}$ under the constraint $\mathcal{C}=0$. As we shall see in the following, the function $\eta$ turns out to be convex. This easily implies proposition 6.1.

Also, the convexity of $\eta$ implies that the solution of the variational problem, $\bar{S}$, is a delta-function centred at $u=0$, i.e. at $v=\omega / \pi$ (so that $\mathcal{C}$ vanishes).

So it only remains for us to prove that $\eta$ is a convex function of $u$. By direct computation, we get

$$
6 \eta^{\prime}=\frac{\mathrm{d}}{\mathrm{d} u}\left(G^{-1}(u)\right)^{2}=2 G^{-1}(u) \frac{\mathrm{d} G^{-1}(u)}{\mathrm{d} u}=\frac{2 v}{G^{\prime}(v)} .
$$

Hence, it is sufficient to prove that the function $G^{\prime}(v) / v$ is strictly decreasing in the interval $v>\omega / 2 \pi$. Without loss of generality, we set $\omega=1$; then

$$
G(v)=\frac{v \sin (1 / v)}{1-\cos (1 / v)}
$$

Consider a new function

$$
H(v):=\frac{G^{\prime}(v)}{v}=\frac{1+v \sin (1 / v)}{v^{2}(1-\cos (1 / v))}
$$


then

$$
H(v)^{\prime}=\frac{-v-\left(-1+v^{2}\right) \sin (1 / v)+v \cos (1 / v)(1+v \sin (1 / v))}{v^{4}(1-\cos (1 / v))^{2}} .
$$

The denominator of $H^{\prime}$ being positive, we only need to prove that the numerator of $H^{\prime}$ is negative in the interval $v>1 / 2 \pi$.

If we replace $v$ by $1 / x$ and multiply the numerator by $x^{2}$, we find the expression

$$
\begin{aligned}
h(x) & =-x+\left(x^{2}-1\right) \sin (x)+\cos (x)(x+\sin x) \\
& =(\cos x-1)(\sin x+x)+x^{2} \sin x .
\end{aligned}
$$

We need to show that $h(x)<0$ in the interval $x \in(0,2 \pi)$. First of all, $h(0)=h(2 \pi)=0$, and for any $x \in(\pi, 2 \pi)$ the expression is clearly negative.

It only remains for us to prove that $h(x)$ is negative in $(0, \pi]$. By computing the Taylor expansion of $h$ about $h=0$, one finds

$$
h(x)=\sum_{k=3}^{+\infty}(-1)^{k} \frac{2^{2 k}-4 k^{2}}{(2 k+1) !} x^{2 k+1} .
$$

It is easy to prove that for any $x \in(0, \pi]$ this is an alternating series, the absolute values of its terms being strictly decreasing.

The first few terms of the above expansion are

$$
h(x)=-\frac{x^{7}}{180}\left(1-\frac{2}{21} x^{2}+\frac{1}{240} x^{4}-\frac{19}{166320} x^{6}\right)+\mathcal{O}\left(x^{15}\right) .
$$

Therefore,

$$
-\frac{x^{7}}{180}<h(x)<-\frac{x^{7}}{180}\left(1-\frac{2}{21} x^{2}\right)
$$

which implies that $h(x)<0$ for any $x \in(0, \pi]$.

\section{Conclusions and open problems}

The instability of a massive piston in a box filled with an ideal gas that is initially in a mechanical (but not thermal) equilibrium was observed in [CL]. It was found there that sometimes this state rapidly breaks down and then the piston and the gas tend to oscillate in an amazingly regular fashion. Our aim here was to understand this phenomenon by analysing the stability of the HEs that govern the time evolution of the system in an appropriate space-time scaling limit.

We have presented several results of our studies. Section 3 contains our only rigorous (mathematical) theorem, which describes conditions under which the phenomenon in question does not occur in the HE. Section 4 presents a semi-heuristic 'perturbative' analysis of the HE that gives a plausible (but not rigorous) criterion (4.9) for the instability of the piston dynamics. This criterion amounts to solving the highly nonlinear equation (4.9), which presents a difficult problem by itself. In fact, in section 5 we try to apply this criterion to a particularly simple family of density functions (5.1) and only obtain partial results. We could not analyse the entire family, but our criterion did yield densities of both sorts: those for which the piston is stable and those for which it is unstable. The densities of both types alternate in a very intricate manner. The conclusions of section 5, though derived heuristically, are in good agreement with computer simulations of the HEs. 
Lastly, a related but separate question is discussed in section 6-what may be the longterm behaviour of oscillations of the piston in the case where the HEs are unstable? We conjecture that there are saddle-type periodic solutions in the phase space that temporarily attract typical unstable trajectories; the latter stay close to those periodic solutions for a long time but eventually spin off to chaotic regions in the phase space. In section 6 we construct approximate periodic solutions using heuristic arguments similar to the perturbative analysis in section 4.

We are clearly far from a complete understanding of the phenomenon in question. Here we only obtain some partial results and outline possible approaches to the problem that require further studies. Indeed, this paper raises more open questions than it answers, and we hope the work in this direction continues.

One possible extension of our studies is to describe the original mechanical system by HEs with an added fluctuation term. This is a result of the granularity of the particle system and should be of $\mathrm{O}\left(N^{-\alpha}\right)$ with some $\alpha>0$. Such fluctuation terms may be derived as a high order corrections to the HEs (see [S]). With such a hydrodynamic fluctuation term, stable stationary solutions of the HEs will remain close to the stationary solutions without fluctuations for a long time, but eventually they will slowly drift away to a thermal equilibrium, i.e. they will behave similar to the particle system.

Concerning the HE discussed in this work, we would like to know if they have periodic or asymptotically periodic solutions and determine their period, amplitude, and structure.

\section{Acknowledgments}

We thank E Presutti and Ya Sinai for their contributions to the early stages of this work, C Marchioro, M Pulvirenti, and N Simanyi for useful discussions. We also thank Weinan E, Rick Falk, and Michael Vogelius for helpful suggestions about the numerical solution of the HEs. We are particularly indebted to O Costin for his extension of our results in section 5 . EC was partially supported by MIUR GNFM and by the European network HYKE, contract HPRN-CT-2002-00282. NC was partially supported by NSF grant DMS-0098788. JLL was partially supported by NSF grant DMR-9813268 and by Air Force grant F49620-01-0154. This work was started when the authors stayed at the Institute for Advanced Study with partial support by NSF grant DMS-9729992.

\section{References}

[CLS1] Chernov N, Lebowitz J L and Sinai Ya 2002 Dynamic of a massive piston in an ideal gas Russ. Math. Surveys 57 1-84

[CLS2] Chernov N, Lebowitz J L and Sinai Ya 2002 Scaling dynamic of a massive piston in a cube filled with ideal gas: exact results J. Stat. Phys. 109 529-48

[CL] Chernov N and Lebowitz J L 2002 Dynamics of a massive piston in an ideal gas: oscillatory motion and approach to equilibrium J. Stat. Phys. 109 507-27

[C] Costin O private communication

[Do] Douady R 1988 PhD Thesis Ecole Polytechnique

[G] Gruber Ch 1999 Thermodynamics of systems with internal adiabatic constraints: time evolution of the adiabatic piston Eur. J. Phys. 20 259-66

[GF] Gruber Ch and Frachebourg L 1999 On the adiabatic properties of a stochastic adiabatic wall: evolution, stationary non-equilibrium, and equilibrium states Phys. A 272 392-428

[GP] Gruber Ch and Piasecki J 1999 Stationary motion of the adiabatic piston Physica A 268 412-23

[H] Holley R 1971 The motion of a heavy particle in an infinite one dimensional gas of hard spheres Z. Wahrschein. verw. Geb. 17 181-219 
[KBM] Kestemont E, Van den Broeck C and Mansour M 2000 The 'adiabatic' piston: and yet it moves Europhys. Lett. 49 143-9

[LL] Laederich S and Levi M 1991 Invariant curves and time-dependent potentials Ergod. Theory Dynam. Sys. 11 365-78

[LPS] Lebowitz J L, Piasecki J and Sinai Ya 2000 Scaling dynamics of a massive piston in an ideal gas Hard Ball Systems and the Lorentz Gas: Encyclopedia of Mathematical Sciences vol 101 (Berlin: Springer) pp 217-27

[Li] Lieb E 1999 Some problems in statistical mechanics that I would like to see solved Physica A 263 491-9

[MP] Marchioro C and Pulvirenti M 1986 A note on the stability of a spatially symmetric Vlasov-Poisson flow Math. Methods Appl. Sci. 8 284-8

[P] Penrose O 1960 Electrostatic instabilities of a uniform non-Maxwellian plasma Phys. Fluids 3 258-65

[PG] Piasecki J and Gruber Ch 1999 From the adiabatic piston to macroscopic motion induced by fluctuations Physica A 265 463-72

[S] Spohn H 1991 Large Scale Dynamics of Interacting Particles (Berlin: Springer) 